Homology, Homotopy and Applications, vol.9(1), 2007, pp.331-366

\title{
ON THE 2-ADIC $K$-LOCALIZATIONS OF $H$-SPACES
}

\author{
A.K. BOUSFIELD
}

(communicated by Donald M. Davis)

\begin{abstract}
We determine the 2-adic $K$-localizations for a large class of $H$-spaces and related spaces. As in the odd primary case, these localizations are expressed as fibers of maps between specified infinite loop spaces, allowing us to approach the 2 -primary $v_{1}$-periodic homotopy groups of our spaces. The present $v_{1}$-periodic results have been applied very successfully to simply-connected compact Lie groups by Davis, using knowledge of the complex, real, and quaternionic representations of the groups. We also functorially determine the united 2-adic $K$-cohomology algebras (including the 2-adic $K O$-cohomology algebras) for all simply-connected compact Lie groups in terms of their representation theories, and we show the existence of spaces realizing a wide class of united 2-adic $K$-cohomology algebras with specified operations.
\end{abstract}

\section{Introduction}

In [20], Mahowald and Thompson determined the $p$-adic $K$-localizations of the odd spheres at an arbitrary prime $p$, expressing these localizations as homotopy fibers of maps between specified infinite loop spaces. Then, working at an odd prime $p$ in [8], we generalized this result to give the $p$-adic $K$-localizations for a large class of $H$-spaces and related spaces. In the present paper, we obtain similar results for 2adic $K$-localizations of such spaces, using our preparatory work in [10] and [11]. By a 2-adic $K$-localization, we mean a $K / 2_{*}$-localization (see [2], [3]), which is the same as a $K^{*}\left(-; \hat{\mathbb{Z}}_{2}\right)$-localization, since the $K / 2_{*}$-equivalences of spaces or spectra are the same as the $K^{*}\left(-; \hat{\mathbb{Z}}_{2}\right)$-equivalences. Our localization results in this paper will apply to many (but not all) simply-connected finite $H$-spaces and to related spaces such as the spheres $S^{4 k-1}$ for $k \geqslant 1$. We show that these results allow computations of the $v_{1}$-periodic homotopy groups (see $[\mathbf{1 3}],[\mathbf{1 5}]$ ) of our spaces from their united 2-adic $K$-cohomologies, and thus allow computations of the $v_{1}$-periodic homotopy groups for a large class of simply-connected compact Lie groups from their complex, real, and quaternionic representation theories. The present results will be extended in a

Received December 18, 2006, revised January 20, 2007; published on March 6, 2007.

2000 Mathematics Subject Classification: 55N15, 55P60, 55Q51, 55S25.

Key words and phrases: $K$-localizations, $v_{1}$-periodic homotopy, 2 -adic $K$-theory, united $K$-theory, compact Lie groups.

Copyright (c) 2007, International Press. Permission to copy for private use granted. 
subsequent paper to cover the remaining simply-connected compact Lie groups and various spaces related to the remaining odd spheres. This work has been applied very successfully by Davis [14] to complete his 13-year program (with Bendersky) of calculating the $v_{1}$-periodic homotopy groups of all simply-connected compact Lie groups, and has also been applied by Bendersky, Davis, and Mahowald [1].

Throughout this paper, we work at the prime 2 and rely on the united 2-adic $K$-cohomology

$$
K_{C R}^{*}\left(X ; \hat{\mathbb{Z}}_{2}\right)=\left\{K^{*}\left(X ; \hat{\mathbb{Z}}_{2}\right), K O^{*}\left(X ; \hat{\mathbb{Z}}_{2}\right)\right\}
$$

of a space or spectrum $X$ as in [10]. This combines the usual periodic cohomologies with certain operations between them, such as complexification and realification. For our $H$-spaces and related spaces $X$, the cohomology $K_{C R}^{*}\left(X ; \hat{\mathbb{Z}}_{2}\right)$ is essentially determined by the 2-adic Adams $\Delta$-module

$$
\widetilde{K}_{\Delta}^{-1}\left(X ; \hat{\mathbb{Z}}_{2}\right)=\left\{\widetilde{K}^{-1}\left(X ; \hat{\mathbb{Z}}_{2}\right), \widetilde{K O}^{-1}\left(X ; \hat{\mathbb{Z}}_{2}\right), \widetilde{K O}^{-5}\left(X ; \hat{\mathbb{Z}}_{2}\right)\right\}
$$

which combines the specified cohomologies with the additive operations among them (see Definition 6.1). In fact, for most simply-connected finite $H$-spaces $X$, we expect to have an isomorphism $K_{C R}^{*}\left(X ; \hat{\mathbb{Z}}_{2}\right) \cong \hat{L}(M)$ where $M=\left\{M_{C}, M_{R}, M_{H}\right\}$ is the submodule of primitives in $\widetilde{K}_{\Delta}^{-1}\left(X ; \hat{\mathbb{Z}}_{2}\right)$ and where $\hat{L}$ is a functor that we introduce in Lemma 4.5, extending the 2 -adic exterior algebra functor on complex components. For a simply-connected compact Lie group $G$, the required 2-adic Adams $\Delta$-module may be obtained as the indecomposables $\hat{Q} R_{\Delta} G=\left\{\hat{Q} R G, \hat{Q} R_{R} G, \hat{Q} R_{H} G\right\}$ of the complex, real, and quaternionic representation $\operatorname{ring} R_{\Delta} G=\left\{R G, R_{R} G, R_{H} G\right\}$ (see Definition 10.1), and we have:

Theorem 1.1. For a simply-connected compact Lie group $G$, there is a natural isomorphism $K_{C R}^{*}\left(G ; \hat{\mathbb{Z}}_{2}\right) \cong \hat{L}\left(\hat{Q} R_{\Delta} G\right)$ of algebras.

This will follow from Theorem 10.3. It extends results of Hodgkin [17], Seymour [23], Minami [21], and others on $K^{*}\left(G ; \hat{\mathbb{Z}}_{2}\right)$ and $K O^{*}\left(G ; \hat{\mathbb{Z}}_{2}\right)$. Our main result on $K / 2_{*}$-localizations will apply to a space $X$ with $K_{C R}^{*}\left(X ; \hat{\mathbb{Z}}_{2}\right) \cong \hat{L} M$ for a 2-adic Adams $\Delta$-module $M$ that is strong (see Definition 7.11). This technical algebraic condition seems relatively mild and holds for $\hat{Q} R_{\Delta} G$ when $G$ is a simply-connected compact simple Lie group other than $E_{6}$ or $\operatorname{Spin}(4 k+2)$ with $k$ not a 2 -power by work of Davis (see Lemma 10.5). For a strong 2-adic Adams $\Delta$-module $M$, we obtain two stable 2-adic Adams $\Delta$-modules $\bar{M}=\left\{\bar{M}_{C}, \bar{M}_{R}, \bar{M}_{H}\right\}$ and $\bar{\rho} \bar{M}=\left\{\bar{M}_{C}, \bar{M}_{R}+\right.$ $\left.\bar{M}_{H}, \bar{M}_{R} \cap \bar{M}_{H}\right\}$ where $\bar{M}_{C}=M_{C}, \bar{M}_{R}=\operatorname{im}\left(M_{R} \rightarrow M_{C}\right)$, and $\bar{M}_{H}=\operatorname{im}\left(M_{H} \rightarrow\right.$ $\left.M_{C}\right)$; and we obtain two corresponding $K / 2_{*}$-local spectra $\mathcal{E} \bar{M}$ and $\mathcal{E} \bar{\rho} \bar{M}$ such that $K_{\Delta}^{-1}\left(\mathcal{E} \bar{M} ; \hat{\mathbb{Z}}_{2}\right)=\bar{M}, K^{0}\left(\mathcal{E} \bar{M} ; \hat{\mathbb{Z}}_{2}\right)=0, K_{\Delta}^{-1}\left(\mathcal{E} \bar{\rho} \bar{M} ; \hat{\mathbb{Z}}_{2}\right)=\bar{\rho} \bar{M}$, and $K^{0}\left(\mathcal{E} \bar{\rho} \bar{M} ; \hat{\mathbb{Z}}_{2}\right)=$ 0 (see Definition 8.1 ). Stated briefly, our main localization result is:

Theorem 1.2. If $X$ is a connected space with $K_{C R}^{*}\left(X ; \hat{\mathbb{Z}}_{2}\right) \cong \hat{L} M$ for a strong 2adic Adams $\Delta$-module $M$, then its $K / 2_{*}$-localization $X_{K / 2}$ is the homotopy fiber of a map from $\Omega^{\infty} \mathcal{E} \bar{M}$ to $\Omega^{\infty} \mathcal{E} \bar{\rho} \bar{M}$ with low dimensional modifications.

This will follow from Theorem 8.6. It will apply to simply-connected compact simple Lie groups with the above-mentioned exceptions, and it should apply to many 
other simply-connected finite $H$-spaces and related spaces; in fact, there must exist a great diversity of spaces with the required united 2 -adic $K$-cohomology algebras by:

Theorem 1.3. For each strong 2-adic Adams $\Delta$-module $M$, there exists a simplyconnected space $X$ with $K_{C R}^{*}\left(X ; \hat{\mathbb{Z}}_{2}\right) \cong \hat{L} M$.

This will follow from Theorem 8.5. For our spaces $X$, we also obtain results on the 2-primary $v_{1}$-periodic homotopy groups $v_{1}^{-1} \pi_{*} X$, which are naturally isomorphic to stable homotopy groups $\pi_{*} \tau_{2} \Phi_{1} X$, where $\tau_{2} \Phi_{1} X$ is the 2-torsion part of the spectrum $\Phi_{1} X$ obtained using the $v_{1}$-stabilization functor $\Phi_{1}$ constructed in $[\mathbf{4}],[\mathbf{9}],[\mathbf{1 6}]$, and [18]. From this standpoint, the homotopy $v_{1}^{-1} \pi_{*} X$ is essentially determined by the cohomology $K O^{*}\left(\Phi_{1} X ; \hat{\mathbb{Z}}_{2}\right)$, since there is an exact sequence

$$
\begin{gathered}
\cdots \longrightarrow K O^{n-3}\left(\Phi_{1} X ; \hat{\mathbb{Z}}_{2}\right) \stackrel{\psi^{3}-9}{\longrightarrow} K O^{n-3}\left(\Phi_{1} X ; \hat{\mathbb{Z}}_{2}\right) \longrightarrow\left(v_{1}^{-1} \pi_{n} X\right)^{\#} \\
\longrightarrow K O^{n-2}\left(\Phi_{1} X ; \hat{\mathbb{Z}}_{2}\right) \stackrel{\psi^{3}-9}{\longrightarrow} K O^{n-2}\left(\Phi_{1} X ; \hat{\mathbb{Z}}_{2}\right) \longrightarrow \cdots
\end{gathered}
$$

where $(-)^{\#}$ gives the Pontrjagin dual (see Theorem 9.2). A space $X$ is called $K / 2_{*}$ durable when the $K / 2_{*}$-localization induces an isomorphism $v_{1}^{-1} \pi_{*} X \cong v_{1}^{-1} \pi_{*} X_{K / 2}$ or equivalently $\Phi_{1} X \simeq \Phi_{1} X_{K / 2}$. This condition holds for all connected $H$-spaces (and many other spaces), and our $K / 2_{*}$-localization result implies:

Theorem 1.4. If $X$ is a connected $K / 2_{*}$-durable space (e.g. H-space) with $K_{C R}^{*}\left(X ; \hat{\mathbb{Z}}_{2}\right) \cong \hat{L} M$ for a strong 2-adic Adams $\Delta$-module $M$, then there is a (co)fiber sequence of spectra $\Phi_{1} X \rightarrow \mathcal{E} \bar{M} \rightarrow \mathcal{E} \bar{\rho} \bar{M}$ with a KO* $\left(-; \hat{\mathbb{Z}}_{2}\right)$ cohomology exact sequence

$$
\begin{aligned}
& 0 \longrightarrow K O^{-8}\left(\Phi_{1} X ; \hat{\mathbb{Z}}_{2}\right) \longrightarrow \bar{M}_{C} /\left(\bar{M}_{R}+\bar{M}_{H}\right) \stackrel{\lambda^{2}}{\longrightarrow} \bar{M}_{C} / \bar{M}_{R} \longrightarrow K O^{-7}\left(\Phi_{1} X ; \hat{\mathbb{Z}}_{2}\right) \\
& \longrightarrow 0 \longrightarrow \bar{M}_{H} /\left(\bar{M}_{R} \cap \bar{M}_{H}\right) \longrightarrow K O^{-6}\left(\Phi_{1} X ; \hat{\mathbb{Z}}_{2}\right) \longrightarrow \bar{M}_{R} \cap \bar{M}_{H} \stackrel{\lambda^{2}}{\longrightarrow} \bar{M}_{H} \longrightarrow \\
& K O^{-5}\left(\Phi_{1} X ; \hat{\mathbb{Z}}_{2}\right) \longrightarrow 0 \longrightarrow 0 \longrightarrow K O^{-4}\left(\Phi_{1} X ; \hat{\mathbb{Z}}_{2}\right) \longrightarrow \bar{M}_{C} /\left(\bar{M}_{R} \cap \bar{M}_{H}\right) \stackrel{\lambda^{2}}{\longrightarrow} \\
& \bar{M}_{C} / \bar{M}_{H} \longrightarrow K O^{-3}\left(\Phi_{1} X ; \hat{\mathbb{Z}}_{2}\right) \longrightarrow\left(\bar{M}_{R}+\bar{M}_{H}\right) /\left(\bar{M}_{R} \cap \bar{M}_{H}\right) \stackrel{\lambda^{2}}{\longrightarrow} \\
& K O^{-2}\left(\Phi_{1} X ; \hat{\mathbb{Z}}_{2}\right) \longrightarrow \bar{M}_{R}+\bar{M}_{H} \stackrel{\lambda^{2}}{\longrightarrow} \bar{M}_{R} \longrightarrow K O^{-1}\left(\Phi_{1} X ; \hat{\mathbb{Z}}_{2}\right) \longrightarrow 0 \text {. }
\end{aligned}
$$

This will follow from Theorem 9.5. It allows effective computations of 2-primary $v_{1}$-periodic homotopy groups as shown by Davis [14], and its complex analogue implies that our spaces $X$ are usually $\widehat{K} \Phi_{1}$-good, which means that $\hat{Q} K^{n}\left(X ; \hat{\mathbb{Z}}_{2}\right) / \lambda^{2}$ $\cong K^{n}\left(\Phi_{1} X ; \hat{\mathbb{Z}}_{2}\right)$ for $n=-1,0$.

Theorem 1.5. If $X$ is as in Theorem 1.4 with $\lambda^{2}: M_{C} \rightarrow M_{C}$ monic, then $X$ is $\widehat{K} \Phi_{1}-$ good.

This will follow from Theorem 9.7. It will be used in a subsequent paper to show that all simply-connected compact Lie groups (and many other spaces) are $\widehat{K} \Phi_{1}$-good, which is useful because the $v_{1}$-periodic homotopy groups of $\widehat{K} \Phi_{1}$-good spaces are often accessible by $[\mathbf{1 0}]$, even when our $K / 2_{*}$-localization theorems do not 
apply. From the perspective of [10], the present work verifies important examples of $\widehat{K} \Phi_{1}$-good spaces beyond the odd spheres.

Throughout the paper, spaces and spectra will belong to the usual pointed simplicial or CW homotopy categories. To provide a suitably precise setting for our main theorems and proofs, we must devote considerable attention to developing the algebraic infrastructure of united 2-adic $K$-cohomology theory. The paper is divided into the following sections:

\section{Introduction}

2. The united 2-adic $K$-cohomologies of spectra and spaces

3. The 2-adic $\phi C R$-algebras

4. The universal 2-adic $\phi C R$-algebra functor $\hat{L}$

5. Stable 2-adic Adams operations and $K / 2_{*}$-local spectra

6. On the united 2-adic $K$-cohomologies of infinite loop spaces

7. Strong 2-adic Adams $\Delta$-modules

8. On the $K / 2_{*}$-localizations of our spaces

9. On the $v_{1}$-periodic homotopy groups of our spaces

10. Applications to simply-connected compact Lie groups

11. Proofs of basic lemmas for $\hat{L}$

12. Proof of the Bott exactness lemma for $\hat{L}$

13. Proofs for regular modules

14. Proof of the realizability theorem for $\hat{L} M$

Although we have long been interested in the $K$-localizations and $v_{1}$-periodic homotopy groups of spaces, we were prompted to develop the present results by Martin Bendersky and Don Davis. We thank them for their questions and comments.

\section{The united 2-adic $K$-cohomologies of spectra and spaces}

We now consider the united 2-adic $K$-cohomologies

$$
K_{C R}^{*}\left(X ; \hat{\mathbb{Z}}_{2}\right)=\left\{K^{*}\left(X ; \hat{\mathbb{Z}}_{2}\right), K O^{*}\left(X ; \hat{\mathbb{Z}}_{2}\right)\right\}
$$

of spectra and spaces $X$, focusing on their basic structures as 2-adic $C R$-modules or $C R$-algebras. We first recall:

Definition 2.1 (The 2-adic $C R$-modules). By a 2-adic $C R$-module, we mean a $C R$ module over the category of 2-profinite abelian groups (see [10, 4.1]). Thus, a 2-adic $C R$-module $M=\left\{M_{C}, M_{R}\right\}$ consists of $\mathbb{Z}$-graded 2-profinite abelian groups $M_{C}$ and $M_{R}$ with continuous additive operations

$$
\begin{array}{clrl}
B: M_{C}^{*} \cong M_{C}^{*-2}, & t: M_{C}^{*} \cong M_{C}^{*}, & B_{R}: M_{R}^{*} \cong M_{R}^{*-8}, \\
\eta: M_{R}^{*} \rightarrow M_{R}^{*-1}, & c: M_{R}^{*} \rightarrow M_{C}^{*}, & r: M_{C}^{*} \rightarrow M_{R}^{*},
\end{array}
$$


satisfying the relations

$$
\begin{aligned}
& 2 \eta=0, \quad \eta^{3}=0, \quad \eta B_{R}=B_{R} \eta, \quad \eta r=0, \quad c \eta=0, \\
& t^{2}=1, \quad t B=-B t, \quad r t=r, \quad t c=c, \quad c B_{R}=B^{4} c, \\
& r B^{4}=B_{R} r, \quad c r=1+t, \quad r c=2, \quad r B c=\eta^{2}, \quad r B^{-1} c=0 .
\end{aligned}
$$

For $z \in M_{C}^{*}$ and $x \in M_{R}^{*}$, the elements $t z \in M_{C}^{*}$ and $r B^{2} c x \in M_{R}^{*}$ are sometimes written as $z^{*}\left(\right.$ or $\left.\psi^{-1} z\right)$ and $\xi x$. For a spectrum or space $X$, the united 2-adic $K$-cohomology

$$
K_{C R}^{*}\left(X ; \hat{\mathbb{Z}}_{2}\right)=\left\{K^{*}\left(X ; \hat{\mathbb{Z}}_{2}\right), K O^{*}\left(X ; \hat{\mathbb{Z}}_{2}\right)\right\}
$$

has a natural 2-adic $C R$-module structure with the usual periodicities $B: K^{*}\left(X ; \hat{\mathbb{Z}}_{2}\right)$ $\cong K^{*-2}\left(X ; \hat{\mathbb{Z}}_{2}\right)$, and $B_{R}: K O^{*}\left(X ; \hat{\mathbb{Z}}_{2}\right) \cong K O^{*-8}\left(X ; \hat{\mathbb{Z}}_{2}\right)$, conjugation $t: K^{*}\left(X ; \hat{\mathbb{Z}}_{2}\right)$ $\cong K^{*}\left(X ; \hat{\mathbb{Z}}_{2}\right)$, Hopf operation $\eta: K O^{*}\left(X ; \hat{\mathbb{Z}}_{2}\right) \rightarrow K O^{*-1}\left(X ; \hat{\mathbb{Z}}_{2}\right)$, complexification $c: K O^{*}\left(X ; \hat{\mathbb{Z}}_{2}\right) \rightarrow K^{*}\left(X ; \hat{\mathbb{Z}}_{2}\right)$, and realification $r: K^{*}\left(X ; \hat{\mathbb{Z}}_{2}\right) \rightarrow K O^{*}\left(X ; \hat{\mathbb{Z}}_{2}\right)$.

Definition 2.2 (Bott exactness). As in [10,4.1], we say that a 2-adic $C R$-module $M$ is Bott exact when the Bott sequence

$$
\cdots \longrightarrow M_{R}^{*+1} \stackrel{\eta}{\longrightarrow} M_{R}^{*} \stackrel{c}{\longrightarrow} M_{C}^{*} \stackrel{r B^{-1}}{\longrightarrow} M_{R}^{*+2} \stackrel{\eta}{\longrightarrow} \cdots
$$

is exact, and we note that the 2-adic $C R$-module $K_{C R}^{*}\left(X ; \hat{\mathbb{Z}}_{2}\right)$ is always Bott exact for a spectrum or space $X$. To compare $C R$-modules, we shall often use:

Lemma 2.3. For Bott exact 2-adic CR-modules $M$ and $N$, a map $f: M \rightarrow N$ is an isomorphism if and only if $f: M_{C} \rightarrow N_{C}$ is an isomorphism.

Proof. For the "if" part, we treat the Bott sequences of $M$ and $N$ as exact couples, and we note that $f$ induces an isomorphism of the associated spectral sequences since $f: M_{C} \cong N_{C}$. Using the map of second derived couples with $f: M_{C}^{(2)} \cong N_{C}^{(2)}$, we easily see that $f: \eta^{2} M_{R} \cong \eta^{2} N_{R}$; then using the map of first derived couples with $f: M_{C}^{(1)} \cong N_{C}^{(1)}$, we easily see that $f: \eta M_{R} \cong \eta N_{R}$; and finally using the original map of exact couples, we easily see that $f: M_{R} \cong N_{R}$.

Definition 2.4 (The free 2-adic $C R$-modules). For each integer $n$ and $L=C, R$, there is a monogenic free 2-adic CR-module $F^{L}(g, n)$ on a generator $g \in F^{L}(g, n)_{L}^{n}$ having the universal property that, for each 2-adic $C R$-module $\mathrm{M}$ and $y \in M_{L}^{n}$, there is a unique map $f: F^{L}(g, n) \rightarrow M$ with $f(g)=y$. The 2-adic $C R$ modules $F^{C}(g, n)$ and $F^{R}(g, n)$ are given more explicitly by

$$
\begin{aligned}
& F^{C}(g, n)_{C}^{n-2 i}=\hat{\mathbb{Z}}_{2} \oplus \hat{\mathbb{Z}}_{2}=\left\langle B^{i} g\right\rangle \oplus\left\langle B^{i} g^{*}\right\rangle, F^{C}(g, n)_{C}^{n-2 i-1}=0, \\
& F^{C}(g, n)_{R}^{n-2 i}=\hat{\mathbb{Z}}_{2}=\left\langle r B^{i} g\right\rangle, \quad F^{C}(g, n)_{R}^{n-2 i-1}=0, \\
& F^{R}(g, n)_{C}^{n-2 i}=\hat{\mathbb{Z}}_{2}=\left\langle B^{i} c g\right\rangle, \quad F^{R}(g, n)_{C}^{n-2 i-1}=0, \\
& F^{R}(g, n)_{R}^{n-8 i}=\hat{\mathbb{Z}}_{2}=\left\langle B_{R}^{i} g\right\rangle, \quad F^{R}(g, n)_{R}^{n-8 i-1}=\mathbb{Z} / 2=\left\langle B_{R}^{i} \eta g\right\rangle, \\
& F^{R}(g, n)_{R}^{n-8 i-2}=\mathbb{Z} / 2=\left\langle B_{R}^{i} \eta^{2} g\right\rangle, \quad F^{R}(g, n)_{R}^{n-8 i-4}=\hat{\mathbb{Z}}_{2}=\left\langle B_{R}^{i} \xi g\right\rangle, \\
& F^{R}(g, n)_{R}^{n-8 i-k}=0 \text { for } k=3,5,6,7 \text {. }
\end{aligned}
$$

We note that $F^{C}(g, n)$ and $F^{R}(g, n)$ are Bott exact for all $n$. In general, a free 
2-adic $C R$-module on a finite set of generators may be constructed as a direct sum of the corresponding monogenic free 2 -adic $C R$-modules. To test for this freeness, we may use:

Lemma 2.5. For a Bott exact 2-adic CR-module $M$ (e.g. for some $M=$ $\left.K_{C R}^{*}\left(X ; \hat{\mathbb{Z}}_{2}\right)\right)$, if $M_{C}^{*}$ is a free module over $\hat{K}^{*}=\hat{\mathbb{Z}}_{2}\left[B, B^{-1}\right]$ on the generators $\left\{c a_{i}\right\}_{i} \amalg\left\{b_{j}\right\}_{j} \amalg\left\{b_{j}^{*}\right\}_{j}$ for finite sets of elements $\left\{a_{i}\right\}_{i}$ in $M_{R}^{*}$ and $\left\{b_{j}\right\}_{j}$ in $M_{C}^{*}$, then $M$ is a free 2-adic CR-module on the generators $\left\{a_{i}\right\}_{i}$ and $\left\{b_{j}\right\}_{j}$.

Proof. The canonical map to $M$ from the specified 2-adic $C R$-module is an isomorphism by Lemma 2.3 .

To describe the multiplicative structure of $K_{C R}^{*}\left(X ; \hat{\mathbb{Z}}_{2}\right)$ for a space $X$, we introduce:

Definition 2.6 (The 2-adic $C R$-algebras). By a 2-adic $C R$-algebra $A=\left\{A_{C}, A_{R}\right\}$, we mean a 2-adic $C R$-module with continuous bilinear multiplications $A_{L}^{m} \times A_{L}^{n} \rightarrow$ $A_{L}^{m+n}$ and elements $1 \in A_{L}^{0}$ for $m, n \in \mathbb{Z}$ and $L=C, R$ such that:

(i) the multiplication in $A_{C}^{*}$ and $A_{R}^{*}$ is graded commutative and associative with identity 1 ;

(ii) $B(z w)=(B z) w=z(B w)$ and $(z w)^{*}=z^{*} w^{*}$ for $z \in A_{C}^{m}$ and $w \in A_{C}^{n}$;

(iii) $B_{R}(x y)=\left(B_{R} x\right) y=x\left(B_{R} y\right), \eta(x y)=(\eta x) y=x(\eta y)$, and $\xi(x y)=(\xi x) y=$ $x(\xi y)$ for $x \in A_{R}^{m}$ and $y \in A_{R}^{n}$;

(iv) $c 1=1$ and $c(x y)=(c x)(c y)$ for $x \in A_{R}^{m}$ and $y \in A_{R}^{n}$;

(v) $r((c x) z)=x(r z)$ and $r(z(c x))=(r z) x$ for $x \in A_{R}^{m}$ and $z \in A_{C}^{n}$.

Equivalently, a 2-adic $C R$-algebra $A$ consists of a 2-adic $C R$-module with a commutative associative multiplication $A \hat{\otimes}_{C R} A \rightarrow A$ with identity $\underline{e} \rightarrow A$ for $\underline{e}=F^{R}(1,0)$ $\cong K_{C R}^{*}\left(\mathrm{pt} ; \hat{\mathbb{Z}}_{2}\right)$, where $\hat{\otimes}_{C R}$ is the (symmetric monoidal) complete tensor product for 2-adic $C R$-modules $[\mathbf{1 1}, 2.6]$.

Definition 2.7 (Augmentations and nilpotency). For a 2-adic $C R$-algebra $A$, an augmentation is a map $A \rightarrow \underline{e}$ of 2 -adic $C R$-algebras which is left inverse to the identity $\underline{e} \rightarrow A$. When $A$ is augmented, we let $\tilde{A}=\left\{\tilde{A}_{C}, \tilde{A}_{R}\right\}$ denote the augmentation ideal, and for $m \geqslant 1$ we let $\tilde{A}(m)$ denote the $m$-th power of $\tilde{A}$ given by the image of the $m$-fold product $\tilde{A} \hat{\otimes}_{C R} \cdots \hat{\otimes}_{C R} \tilde{A} \rightarrow \tilde{A}$. Thus, $\tilde{A}(m)_{C}$ is the image of the $m$-fold product $\tilde{A}_{C}^{*} \hat{\otimes} \cdots \hat{\otimes} \tilde{A}_{C}^{*} \rightarrow \tilde{A}_{C}^{*}$, while $\tilde{A}(m)_{R}$ is the image of the $m$-fold product $\tilde{A}_{R}^{*} \hat{\otimes} \cdots \hat{\otimes} \tilde{A}_{R}^{*} \rightarrow \tilde{A}_{R}^{*}$ plus the realification of $\tilde{A}(m)_{C}$. The indecomposables of A are given by the 2-adic $C R$-module $\hat{Q} A=\tilde{A} / \tilde{A}(2)$. We call $A$ nilpotent when $\tilde{A}(m)=0$ for sufficiently large $m$ and call $A$ pro-nilpotent when $\cap_{m} \tilde{A}(m)=0$ or equivalently when $A \cong \lim _{m} A / \tilde{A}(m)$. For a space $X$, the cohomology $K_{C R}^{*}\left(X ; \hat{\mathbb{Z}}_{2}\right)$ has a canonical augmentation $K_{C R}^{*}\left(X ; \hat{\mathbb{Z}}_{2}\right) \rightarrow \underline{e}$ induced by the basepoint pt $\subset X$ with the usual augmentation ideal $\widetilde{K}_{C R}^{*}\left(X ; \hat{\mathbb{Z}}_{2}\right)=\left\{\widetilde{K}^{*}\left(X ; \hat{\mathbb{Z}}_{2}\right), \widetilde{K O}^{*}\left(X ; \hat{\mathbb{Z}}_{2}\right)\right\}$. Moreover, when $X$ is connected, the cohomology $K_{C R}^{*}\left(X ; \hat{\mathbb{Z}}_{2}\right)$ is pro-nilpotent since it is the inverse limit of the cohomologies $K_{C R}^{*}\left(X_{\alpha} ; \hat{\mathbb{Z}}_{2}\right)$ for the finite connected subspaces $X_{\alpha} \subset X$, where each $K_{C R}^{*}\left(X_{\alpha} ; \hat{\mathbb{Z}}_{2}\right)$ is nilpotent. 


\section{The 2-adic $\phi C R$-algebras}

To capture some additional features of the 2-adic $C R$-algebras $K_{C R}^{*}\left(X ; \hat{\mathbb{Z}}_{2}\right)$ for spaces $X$, we now introduce the 2 -adic $\phi C R$-algebras. These structures are often surprisingly rigid and will allow us to construct convenient bases for $K_{C R}^{*}\left(X ; \hat{\mathbb{Z}}_{2}\right)$ in some important general cases, for instance, when $X$ is a simply-connected compact Lie group.

Definition 3.1 (The 2-adic $\phi C R$-algebras). By a 2-adic $\phi C R$-algebra $A$, we mean a 2-adic $C R$-algebra with continuous functions $\phi: A_{C}^{0} \rightarrow A_{R}^{0}$ and $\phi: A_{C}^{-1} \rightarrow A_{R}^{0}$ such that:

(i) $c \phi a=a^{*} a$ and $c \phi x=B^{-1} x^{*} x$ for $a \in A_{C}^{0}$ and $x \in A_{C}^{-1}$;

(ii) $\phi(a+b)=\phi a+\phi b+r\left(a^{*} b\right)$ and $\phi(x+y)=\phi x+\phi y+r B^{-1}\left(x^{*} y\right)$ for $a, b \in$ $A_{C}^{0}$ and $x, y \in A_{C}^{-1}$;

(iii) $\phi(a b)=(\phi a)(\phi b), \phi(a x)=(\phi a)(\phi x)$, and $\phi B^{-1}(x y)=(\phi x)(\phi y)$ for $a, b \in A_{C}^{0}$ and $x, y \in A_{C}^{-1}$;

(iv) $\phi(1)=1, \phi(k a)=k^{2} \phi a, \phi\left(a^{*}\right)=\phi a, \phi(k x)=k^{2} \phi x$, and $\phi\left(x^{*}\right)=-\phi x$ for $a \in$ $A_{C}^{0}, x \in A_{C}^{-1}$, and $k \in \hat{\mathbb{Z}}_{2}$.

For convenience, we extend the operation $\phi$ periodically to give $\phi: A_{C}^{2 i} \rightarrow A_{R}^{0}$ and $\phi: A_{C}^{2 i-1} \rightarrow A_{R}^{0}$ with $\phi w=\phi B^{i} w$ for all $i$ and elements $w$. For a space $X$, the cohomology $K_{C R}^{*}\left(X ; \hat{\mathbb{Z}}_{2}\right)$ has a natural 2-adic $\phi C R$-algebra structure with $\phi: K^{*}\left(X ; \hat{\mathbb{Z}}_{2}\right)$ $\rightarrow K O^{0}\left(X ; \hat{\mathbb{Z}}_{2}\right)$ as in $\left[\mathbf{1 1}\right.$, Section 3]. In particular, $\underline{e} \cong K_{C R}^{*}\left(\mathrm{pt} ; \hat{\mathbb{Z}}_{2}\right)$ is a 2 -adic $\phi C R$ algebra with $\phi(k 1)=k^{2} 1$ for $k \in \hat{\mathbb{Z}}_{2}$. For a 2-adic $\phi C R$-algebra $A$, an augmentation is a map $A \rightarrow \underline{e}$ of 2-adic $\phi C R$-algebras which is left inverse to the identity, and we retain the other notation and terminology of Definition 2.7. Thus, for a space $X$, the $\phi C R$-algebra $K_{C R}^{*}\left(X ; \hat{\mathbb{Z}}_{2}\right)$ has a canonical augmentation and is pro-nilpotent whenever $X$ is connected. To capture some other needed features, we introduce:

Definition 3.2 (The special 2-adic $\phi C R$-algebras). A 2-adic $\phi C R$-algebra $A$ is called special when:

(i) $A$ is augmented and pro-nilpotent;

(ii) $z^{2}=0$ for $z \in A_{C}^{n}$ with $n$ odd;

(iii) $y^{2}=0$ for $y \in A_{R}^{n}$ with $n \equiv 1,-3 \bmod 8$;

(iv) $\phi c x=0$ for $x \in A_{R}^{n}$ with $n \equiv-1,-5 \bmod 8$.

For a connected space $X$, the cohomology $K_{C R}^{*}\left(X ; \hat{\mathbb{Z}}_{2}\right)$ is a special 2-adic $\phi C R$ algebra by [11, Section 3].

Definition 3.3 (Simple systems of generators). Let $A$ be a special 2 -adic $\phi C R$ algebra. By a simple system of generators of odd degree for $A$, we mean finite ordered sets of odd-degree elements $\left\{x_{i}\right\}_{i}$ in $\tilde{A}_{R}$ and $\left\{z_{j}\right\}_{j}$ in $\tilde{A}_{C}$ such that $A_{C}$ is an exterior algebra over $\hat{K}^{*}=\hat{\mathbb{Z}}_{2}\left[B, B^{-1}\right]$ on the generators $\left\{c x_{i}\right\}_{i} \amalg\left\{z_{j}\right\}_{j} \amalg\left\{z_{j}^{*}\right\}_{j}$. 
Such a simple system determines associated products

$$
\begin{gathered}
x_{i_{1}} \ldots x_{i_{m}}\left(\phi z_{j_{1}}\right) \ldots\left(\phi z_{j_{n}}\right) \in A_{R} \\
\left(c x_{i_{1}}\right) \ldots\left(c x_{i_{m}}\right)\left(c \phi z_{j_{1}}\right) \ldots\left(c \phi z_{j_{n}}\right) w_{k_{1}} \ldots w_{k_{q}} \in A_{C}
\end{gathered}
$$

where: $i_{1}<\cdots<i_{m}$ with $m \geqslant 0 ; j_{1}<\cdots<j_{n}$ with $n \geqslant 0 ; k_{1}<\cdots<k_{q}$ with $q \geqslant 1$; each $w_{k_{t}}$ is $z_{k_{t}}$ or $z_{k_{t}}^{*}$ with $w_{k_{q}}=z_{k_{q}}$; and $\left\{k_{1}, \ldots, k_{q}\right\}$ is disjoint from $\left\{j_{1}, \ldots, j_{n}\right\}$ in each complex product.

Proposition 3.4. If $A$ is a Bott exact special 2-adic $\phi C R$-algebra with a simple system of generators of odd degree, then $A$ is a free 2-adic CR-module on the associated products.

Proof. This follows by Lemma 2.5 .

When the cohomology $K_{C R}^{*}\left(X ; \hat{\mathbb{Z}}_{2}\right)$ of a connected space $X$ has a simple system of generators of odd degree, this result will determine the 2-adic $C R$-algebra structure of the cohomology, provided that we can compute the squares of the real simple generators of degree $\equiv-1,-5 \bmod 8$, since the squares of the other simple generators and of their $\phi$ 's must vanish. For a simply-connected compact Lie group $G$, we shall see that the cohomology $K_{C R}^{*}\left(G ; \hat{\mathbb{Z}}_{2}\right)$ must always have a simple system of generators of odd degree by Theorem 10.3 below.

\section{The universal 2-adic $\phi C R$-algebra functor $\hat{L}$}

We must now go beyond simple systems of generators and develop functorial descriptions of cohomologies $K_{C R}^{*}\left(X ; \hat{\mathbb{Z}}_{2}\right)$ using universal special 2-adic $\phi C R$ algebras. Our results will apply, for instance, when $X$ is a suitable infinite loop space (Theorem 6.7) or a simply-connected compact Lie group (Theorem 10.3). We start by introducing the algebraic modules that will generate our universal algebras.

Definition 4.1 (The 2-adic $\Delta$-modules). By a 2-adic $\Delta$-module $N=\left\{N_{C}, N_{R}\right.$, $\left.N_{H}\right\}$, we mean a triad of 2-profinite abelian groups $N_{C}, N_{R}$, and $N_{H}$ with continuous additive operations

$$
\begin{gathered}
t: N_{C} \cong N_{C}, \quad c: N_{R} \rightarrow N_{C}, \quad r: N_{C} \rightarrow N_{R} \\
c^{\prime}: N_{H} \rightarrow N_{C}, \quad q: N_{C} \rightarrow N_{H}
\end{gathered}
$$

satisfying the relations

$$
\begin{gathered}
t^{2}=1, \quad c r=1+t, \quad r c=2, \quad t c=c, \quad r t=r, \quad \\
c^{\prime} q=1+t, \quad q c^{\prime}=2, \quad t c^{\prime}=c^{\prime}, \quad q t=q
\end{gathered}
$$

as in $[\mathbf{1 0}, 4.5]$. For $z \in N_{C}$, the element $t z$ is sometimes written as $z^{*}$ or $\psi^{-1} z$. For a 2-adic $C R$-module $N$ and integer $n$, we obtain a 2-adic $\Delta$-module $\Delta^{n} N=$ $\left\{N_{C}^{n}, N_{R}^{n}, N_{R}^{n-4}\right\}$ with $c^{\prime}=B^{-2} c: N_{R}^{n-4} \rightarrow N_{C}^{n}$ and $q=r B^{2}: N_{C}^{n} \rightarrow N_{R}^{n-4}$. In particular, we obtain a 2 -adic $\Delta$-module $K_{\Delta}^{n}\left(X ; \hat{\mathbb{Z}}_{2}\right)=\Delta^{n} K_{C R}^{*}\left(X ; \hat{\mathbb{Z}}_{2}\right)$ for a space $X$. 
We say that a 2-adic $\Delta$-module $N$ is torsion-free when $N_{C}, N_{R}$, and $N_{H}$ are torsionfree, and we say that $N$ is exact when the sequence

$$
\cdots \longrightarrow N_{C} \stackrel{(r, q)}{\longrightarrow} N_{R} \oplus N_{H} \stackrel{c-c^{\prime}}{\longrightarrow} N_{C} \stackrel{1-t}{\longrightarrow} N_{C} \stackrel{(r, q)}{\longrightarrow} N_{R} \oplus N_{H} \longrightarrow \cdots
$$

is exact (see $[\mathbf{1 0}, 4.5])$. It is straightforward to show:

Lemma 4.2. A 2-adic $\Delta$-module $N=\left\{N_{C}, N_{R}, N_{H}\right\}$ is torsion-free and exact if and only if:

(i) $c: N_{R} \rightarrow N_{C}$ and $c^{\prime}: N_{H} \rightarrow N_{C}$ are monic;

(ii) $N_{C}$ is torsion-free with $\operatorname{ker}(1+t)=\operatorname{im}(1-t)$ for $t: N_{C} \rightarrow N_{C}$;

(iii) $c N_{R}+c^{\prime} N_{H}=\operatorname{ker}(1-t)$ and $c N_{R} \cap c^{\prime} N_{H}=\operatorname{im}(1+t)$.

The 2-adic $\Delta$-module

$$
K_{\Delta}^{-1}\left(X ; \hat{\mathbb{Z}}_{2}\right)=\left\{K^{-1}\left(X ; \hat{\mathbb{Z}}_{2}\right), K O^{-1}\left(X ; \hat{\mathbb{Z}}_{2}\right), K O^{-5}\left(X ; \hat{\mathbb{Z}}_{2}\right)\right\}
$$

of a space $X$ has additional operations $\theta$ which we now include in:

Definition 4.3 (The 2-adic $\theta \Delta$-modules). By a 2-adic $\theta \Delta$-module $M=\left\{M_{C}, M_{R}\right.$, $M_{H}$ \}, we mean a 2-adic $\Delta$-module with continuous additive operations $\theta: M_{C} \rightarrow$ $M_{C}, \theta: M_{R} \rightarrow M_{R}$, and $\theta: M_{H} \rightarrow M_{R}$ satisfying the following relations for elements $z \in M_{C}, x \in M_{R}$, and $y \in M_{H}$ :

$$
\theta c x=c \theta x, \quad \theta c^{\prime} y=c \theta y, \quad \theta t z=t \theta z, \quad \theta q z=\theta r z, \quad \theta \theta r z=\theta r \theta z .
$$

In general, $\theta r z$ may differ from $r \theta z$, and we let $\bar{\phi}: M_{C} \rightarrow M_{R}$ be the difference operation with $\bar{\phi} z=\theta r z-r \theta z$ for $z \in M_{C}$. Using the above relations, we easily deduce:

$$
\begin{aligned}
\bar{\phi} c x & =0, & \bar{\phi} c^{\prime} x & =0, \\
2 \bar{\phi} z & =0, & \bar{\phi} t z & =\bar{\phi} z, \\
c \bar{\phi} z & =0, & \theta \bar{\phi} z & =0 .
\end{aligned}
$$

For a space $X$, the cohomology $K_{\Delta}^{-1}\left(X ; \hat{\mathbb{Z}}_{2}\right)$ has a natural 2-adic $\theta \Delta$-module structure by $[\mathbf{1 1}$, Section 3] with the operations

$$
\begin{aligned}
& \theta=-\lambda^{2}: K^{-1}\left(X ; \hat{\mathbb{Z}}_{2}\right) \longrightarrow K^{-1}\left(X ; \hat{\mathbb{Z}}_{2}\right), \\
& \theta=-\lambda^{2}: K O^{-1}\left(X ; \hat{\mathbb{Z}}_{2}\right) \longrightarrow K O^{-1}\left(X ; \hat{\mathbb{Z}}_{2}\right), \\
& \theta=-\lambda^{2}: K O^{-5}\left(X ; \hat{\mathbb{Z}}_{2}\right) \longrightarrow K O^{-1}\left(X ; \hat{\mathbb{Z}}_{2}\right) .
\end{aligned}
$$

Moreover, this structure interacts with the 2-adic $\phi C R$-algebra structure of $K_{C R}^{*}(X$; $\left.\hat{\mathbb{Z}}_{2}\right)$ in several ways.

Lemma 4.4. For a space $X$, we have:

(i) $\eta \phi z=\bar{\phi} z$ for $z \in K^{-1}\left(X ; \hat{\mathbb{Z}}_{2}\right)$;

(ii) $x^{2}=\eta \theta x$ for $x \in K O^{-1}\left(X ; \hat{\mathbb{Z}}_{2}\right)$;

(iii) $y^{2}=B_{R} \eta \theta y$ for $y \in K O^{-5}\left(X ; \hat{\mathbb{Z}}_{2}\right)$.

Proof. This follows from [11, Section 3]. 
We shall take account of these relations in our universal algebras. For a 2-adic $\theta \Delta$-module $M$ and a special 2-adic $\phi C R$-algebra $A$, an admissible map $\alpha: M \rightarrow A$ consists of a 2-adic $\Delta$-module map $\alpha: M \rightarrow \Delta^{-1} \tilde{A}$ such that:

(i) $\eta \phi \alpha z=\alpha \bar{\phi} z$ in $A_{C}^{-1}$ for each $z \in M_{C}$;

(ii) $(\alpha x)^{2}=\eta \alpha \theta x$ in $A_{R}^{-2}$ for each $x \in M_{R}$;

(iii) $(\alpha y)^{2}=B_{R} \eta \alpha \theta y$ in $A_{R}^{-10}$ for each $y \in M_{H}$.

We say that a special 2-adic $\phi C R$-algebra $A$ with an admissible map $\alpha: M \rightarrow A$ is universal if, for each special 2-adic $\phi C R$-algebra $B$ with admissible map $g: M \rightarrow B$, there exists a unique $\phi C R$-algebra map $\bar{g}: A \rightarrow B$ such that $\bar{g} \alpha=g$.

Lemma 4.5. For each 2-adic $\theta \Delta$-module $M$, there exists a universal special 2-adic $\phi C R$-algebra $\hat{L} M$ with admissible map $\alpha: M \rightarrow \hat{L} M$.

This will be proved later in Section 11. By universality, $\hat{L} M$ is unique up to isomorphism and is natural in $M$, so that we have a functor $\hat{L}$ from the category of 2 -adic $\theta \Delta$-modules to the category of special 2 -adic $\phi C R$-algebras. We believe that the $\phi C R$-algebra $\hat{L} M$ can be given canonical operations $\theta$ satisfying all the formulae of [11, Section 3] and that this provides a strengthened version of $\hat{L}$ that is right adjoint to $\Delta^{-1} \widetilde{()}$. However, for simplicity, we rely on the present basic functor $\hat{L}$. We can describe the algebra $(\hat{L} M)_{C}$ explicitly using the 2-adic exterior algebra $\hat{\Lambda} M_{C}$ with $\hat{\Lambda} M_{C}=\lim _{\beta} \hat{\Lambda} M_{C \beta}$ where $M_{C \beta}$ ranges over the finite 2-adic quotients of $M_{C}$ (ignoring $\theta$ ).

Lemma 4.6. For a 2-adic $\theta \Delta$-module $M$, the canonical map $\hat{\Lambda} M_{C} \rightarrow(\hat{L} M)_{C}$ is an algebra isomorphism.

This will be proved later in Section 11. We must impose extra conditions on $M$ to ensure that $\hat{L} M$ is Bott exact and hence topologically relevant.

Definition 4.7 (The robust 2 -adic $\theta \Delta$-modules). We say that a 2 -adic $\theta \Delta$-module $M$ is profinite when it is the inverse limit of an inverse system of finite 2-adic $\theta \Delta$ modules, and we let $M / \bar{\phi}$ denote the 2-adic $\Delta$-module $\left\{M_{C}, M_{R} / \bar{\phi} M_{C}, M_{H}\right\}$. We call $M$ robust when:

(i) $M$ is profinite;

(ii) $M / \bar{\phi}$ is torsion-free and exact;

(iii) $\operatorname{ker} \bar{\phi}=c M_{R}+c^{\prime} M_{H}+2 M_{C}$.

When $M$ is obtained from $K_{\Delta}^{-1}\left(X ; \hat{\mathbb{Z}}_{2}\right)$ for a space $X$, the profiniteness condition will usually hold automatically since $K_{\Delta}^{-1}\left(X ; \hat{\mathbb{Z}}_{2}\right)=\lim _{\alpha, i} K_{\Delta}^{-1}\left(X_{\alpha} ; \hat{\mathbb{Z}}_{2}\right) / 2^{i}$ for the system of finite subcomplexes $X_{\alpha} \subset X$ and $i \geqslant 1$. The following key lemma will be proved later in Section 12.

Lemma 4.8. If $M$ is a robust 2-adic $\theta \Delta$-module, then the special 2-adic $\phi C R$ algebra $\hat{L} M$ is Bott exact; in fact, $\hat{L} M$ is the inverse limit of an inverse system of finitely generated free 2-adic CR-modules.

This leads to a crucial comparison theorem. 
Theorem 4.9. For a connected space $X$ and a robust 2-adic $\theta \Delta$-module $M$, suppose that $g: M \rightarrow \widetilde{K}_{\Delta}^{-1}\left(X ; \hat{\mathbb{Z}}_{2}\right)$ is a 2-adic $\theta \Delta$-module map that induces an isomorphism $\hat{\Lambda} M_{C} \cong K^{*}\left(X ; \hat{\mathbb{Z}}_{2}\right)$. Then $g$ induces an isomorphism $\hat{L} M \cong K_{C R}^{*}\left(X ; \hat{\mathbb{Z}}_{2}\right)$ of special 2-adic $\phi C R$-algebras.

Proof. Since $g$ gives an admissible map $M \rightarrow K_{C R}^{*}\left(X ; \hat{\mathbb{Z}}_{2}\right)$ by Lemma 4.4, the result follows by Lemmas 2.3, 4.6, and 4.8 .

When $M$ is finitely generated in this theorem, we may easily choose a simple system of odd-degree generators (see Definition 3.3) for $K_{C R}^{*}\left(X ; \hat{\mathbb{Z}}_{2}\right)$ from $M_{C}, M_{R}$, and $M_{H}$. However, the present description of $K_{C R}^{*}\left(X ; \hat{\mathbb{Z}}_{2}\right)$ as $\hat{L} M$ is more natural and includes the full multiplicative structure. To check whether such a description is possible for a given space $X$, we may use:

Remark 4.10 (Determination of $M$ from $K_{C R}^{*}\left(X ; \hat{\mathbb{Z}}_{2}\right)$ ). For a connected space $X$, we may take the indecomposables $\hat{Q} K_{C R}^{*}\left(X ; \hat{\mathbb{Z}}_{2}\right)$ as in Definition 2.7 with the operations $\theta$ of Definition 4.3 to produce a 2 -adic $\theta \Delta$-module

$$
\hat{Q} K_{\Delta}^{-1}\left(X ; \hat{\mathbb{Z}}_{2}\right)=\left\{\hat{Q} K^{-1}\left(X ; \hat{\mathbb{Z}}_{2}\right), \hat{Q} K O^{-1}\left(X ; \hat{\mathbb{Z}}_{2}\right), \hat{Q} K O^{-5}\left(X ; \hat{\mathbb{Z}}_{2}\right)\right\}
$$

together with a natural quotient map $\widetilde{K}_{\Delta}^{-1}\left(X ; \hat{\mathbb{Z}}_{2}\right) \rightarrow \hat{Q} K_{\Delta}^{-1}\left(X ; \hat{\mathbb{Z}}_{2}\right)$. Now by Lemma 4.11 below, whenever Theorem 4.9 applies to $X$, there is a canonical isomorphism $M \cong \hat{Q} K_{\Delta}^{-1}\left(X ; \hat{\mathbb{Z}}_{2}\right)$ and the map $g: M \rightarrow \widetilde{K}_{\Delta}^{-1}\left(X ; \hat{\mathbb{Z}}_{2}\right)$ in the theorem corresponds to a splitting of $\widetilde{K}_{\Delta}^{-1}\left(X ; \hat{\mathbb{Z}}_{2}\right) \rightarrow \hat{Q} K_{\Delta}^{-1}\left(X ; \hat{\mathbb{Z}}_{2}\right)$. When $X$ is an $H$-space, we may often obtain the required splitting by mapping $\hat{Q} K_{\Delta}^{-1}\left(X ; \hat{\mathbb{Z}}_{2}\right)$ to the primitives in $\widetilde{K}_{\Delta}^{-1}\left(X ; \hat{\mathbb{Z}}_{2}\right)$. For instance, this applies when $X$ is a suitable infinite loop space or simply-connected compact Lie group (see Theorems 6.7 and 10.3). Finally, we note that the 2 -adic $\theta \Delta$-module $\hat{Q} K_{\Delta}^{-1}\left(X ; \hat{\mathbb{Z}}_{2}\right)$ will automatically be robust by Proposition 3.4 whenever $K_{C R}^{*}\left(X ; \hat{\mathbb{Z}}_{2}\right)$ has a simple system of odd-degree generators with no real generators of degree $\equiv 1,-3 \bmod 8$. We have used:

Lemma 4.11. For a $\theta \Delta$-module $M$, the canonical map $M \rightarrow \Delta^{-1} \hat{Q} \hat{L} M$ is an isomorphism.

This will be proved later in Section 11.

\section{Stable 2-adic Adams operations and $K / 2_{*}$-local spectra}

We now bring stable Adams operations into our united 2-adic $K$-cohomology theory and use this theory to classify the needed $K / 2_{*}$-local spectra. We first recall some terminology from $[8,2.6]$.

Definition 5.1 (The stable 2-adic Adams modules). By a finite stable 2-adic Adams module $A$, we mean a finite abelian 2-group with automorphisms $\psi^{k}: A \cong A$ for the odd $k \in \mathbb{Z}$ such that:

(i) $\psi^{1}=1$ and $\psi^{j} \psi^{k}=\psi^{j k}$ for the odd $j, k \in \mathbb{Z}$;

(ii) when $n$ is sufficiently large, the condition $j \equiv k \bmod 2^{n}$ implies $\psi^{j}=\psi^{k}$. 
By a stable 2-adic Adams module $A$, we mean the topological inverse limit of an inverse system of finite stable 2-adic Adams modules. Such an $A$ has an underlying 2-profinite abelian structure with continuous automorphisms $\psi^{k}: A \cong A$ for the odd $k \in \mathbb{Z}$ (and in fact for $k \in \hat{\mathbb{Z}}_{2}^{\times}$). We note that the operations $\psi^{-1}$ and $\psi^{3}$ on $A$ determine all of the other stable Adams operations $\psi^{k}$ as in [5, 6.4]. Our main examples of stable 2-adic Adams modules are the cohomologies $K^{n}\left(X ; \hat{\mathbb{Z}}_{2}\right)$ and $K O^{n}\left(X ; \hat{\mathbb{Z}}_{2}\right)$ for a spectrum or space $X$ and integer $n$ with the usual stable Adams operations $\psi^{k}$. We let $\hat{\mathcal{A}}$ denote the abelian category of stable 2-adic Adams modules, and for $i \in \mathbb{Z}$ we let $\bar{S}^{i}: \hat{\mathcal{A}} \rightarrow \hat{\mathcal{A}}$ be the functor with $\bar{S}^{i} A$ equal to $A$ as a group but with $\psi^{k}$ on $\bar{S}^{i} A$ equal to $k^{i} \psi^{k}$ on $A$ for the odd $k \in \mathbb{Z}$. We note that $\bar{S}^{i} A=A$ in $\hat{\mathcal{A}}$ for all $i$ when $2 A=0$.

Definition 5.2 (The stable 2-adic Adams CR-modules). By a stable 2-adic Adams $C R$-module $M$, we mean a 2-adic $C R$-module consisting of stable 2-adic Adams modules $\left\{M_{C}^{*}, M_{R}^{*}\right\}$ such that the operations $B: \bar{S} M_{C}^{*} \cong M_{C}^{*-2}, t: M_{C}^{*} \cong M_{C}^{*}$, $B_{R}: \bar{S}^{4} M_{R}^{*} \cong M_{R}^{*-8}, \eta: M_{R}^{*} \rightarrow M_{R}^{*-1}, c: M_{R}^{*} \rightarrow M_{C}^{*}$, and $r: M_{C}^{*} \rightarrow M_{R}^{*}$ are all maps in $\hat{\mathcal{A}}$, where $\psi^{-1}=t$ in $M_{C}^{*}$ and $\psi^{-1}=1$ in $M_{R}^{*}$. For a spectrum or space $X$, the united 2-adic $K$-cohomology

$$
K_{C R}^{*}\left(X ; \hat{\mathbb{Z}}_{2}\right)=\left\{K^{*}\left(X ; \hat{\mathbb{Z}}_{2}\right), K O^{*}\left(X ; \hat{\mathbb{Z}}_{2}\right)\right\}
$$

has a natural stable 2-adic Adams $C R$-module structure with the usual operations.

Definition 5.3 (The stable 2-adic Adams $\Delta$-modules). By a stable 2-adic Adams $\Delta$-module $N$, we mean a 2-adic $\Delta$-module consisting of stable 2-adic Adams modules $\left\{N_{C}, N_{R}, N_{H}\right\}$ such that the operations $t: N_{C} \cong N_{C}, c: N_{R} \rightarrow N_{C}, r: N_{C} \rightarrow N_{R}$, $c^{\prime}: N_{H} \rightarrow N_{C}$, and $q: N_{C} \rightarrow N_{H}$ are all maps in $\hat{\mathcal{A}}$, where $\psi^{-1}=t$ in $N_{C}$ and $\psi^{-1}=1$ in both $N_{R}$ and $N_{H}$. For a stable 2-adic Adams $C R$-module $M$ and integer $n$, we obtain a stable 2 -adic Adams $\Delta$-module

$$
\Delta^{n} M=\left\{M_{C}^{n}, M_{R}^{n}, \bar{S}^{-2} M_{R}^{n-4}\right\}
$$

as in Definition 4.1. Thus, for a spectrum or space $X$ and integer $n$, we now obtain a stable 2-adic Adams $\Delta$-module

$$
K_{\Delta}^{n}\left(X ; \hat{\mathbb{Z}}_{2}\right)=\Delta^{n} K_{C R}^{*}\left(X ; \hat{\mathbb{Z}}_{2}\right)=\left\{K^{n}\left(X ; \hat{\mathbb{Z}}_{2}\right), K O^{n}\left(X ; \hat{\mathbb{Z}}_{2}\right), \bar{S}^{-2} K O^{n-4}\left(X ; \hat{\mathbb{Z}}_{2}\right)\right\}
$$

To give another example, we say that a 2-profinite abelian group $G$ with involution $t: G \cong G$ is positively torsion-free when $G$ is torsion-free with $\operatorname{ker}(1+t)=\operatorname{im}(1-t)$. By [5, Proposition 3.8], this is equivalent to saying that $G$ factors as a (possibly infinite) product of $\hat{\mathbb{Z}}_{2}$ 's with $t=1$ and $\hat{\mathbb{Z}}_{2} \oplus t \hat{\mathbb{Z}}_{2}$ 's. For a positively torsion-free stable 2-adic Adams module $A$, we may use the operation $\psi^{-1}: A \cong A$ to construct a torsion-free exact stable 2-adic Adams $\Delta$-module $\left\{A, A^{+}, A_{+}\right\}$with $A^{+}=\operatorname{ker}(1-$ $\left.\psi^{-1}\right), A_{+}=\operatorname{coker}\left(1-\psi^{-1}\right), t=\psi^{-1}, c=1, r=1+\psi^{-1}, c^{\prime}=1+\psi^{-1}$, and $q=$ 1.

We let $\hat{\mathcal{A C R}}$ (resp. $\hat{\mathcal{A}} \Delta$ ) denote the abelian category of stable 2-adic Adams $C R$ modules (resp. $\Delta$-modules), and we note that the functor $\Delta^{n}: \hat{\mathcal{A C R}} \rightarrow \hat{\mathcal{A}} \Delta$ for $n \in \mathbb{Z}$ has a left adjoint $C R^{n}: \hat{\mathcal{A}} \Delta \rightarrow \hat{\mathcal{A}} \mathcal{C} \mathcal{R}$ with $C R^{n}(N)_{C}^{n}=N_{C}$, with $C R^{n}(N)_{C}^{n-1}=0$, 
and with

$$
C R^{n}(N)_{R}^{n-i}= \begin{cases}N_{R} & \text { for } i=0 \\ N_{R} / r & \text { for } i=1 \\ \bar{S} N_{C} / c^{\prime} & \text { for } i=2 \\ 0 & \text { for } i=3,7 \\ \bar{S}^{2} N_{H} & \text { for } i=4 \\ \bar{S}^{2} N_{H} / q & \text { for } i=5 \\ \bar{S}^{3} N_{C} / c & \text { for } i=6\end{cases}
$$

as in $[\mathbf{1 0}, 4.10]$. We easily see that $C R^{n}(N)$ is Bott exact whenever $N$ is torsion-free and exact. Our next lemma will often allow us to work in the simpler category $\hat{\mathcal{A}} \Delta$ instead of $\hat{\mathcal{A C R}}$.

Lemma 5.4. For $n \in \mathbb{Z}$, the adjoint functors $C R^{n}: \hat{\mathcal{A}} \Delta \rightarrow \hat{\mathcal{A C}} \mathcal{R}$ and $\Delta^{n}: \hat{\mathcal{A}} \mathcal{C} \rightarrow$ $\hat{A} \Delta$ restrict to equivalences between the full subcategories of all torsion-free exact $N \in \hat{\mathcal{A}} \Delta$ and all Bott exact $M \in \hat{\mathcal{A}} \mathcal{C} \mathcal{R}$ with $M_{C}^{n}$ positively torsion-free and $M_{C}^{n-1}=$ 0 .

Proof. For $M \in \hat{A} \mathcal{C} \mathcal{R}$ as above, we see that $\Delta^{n} M$ is a torsion-free exact $\Delta$-module by $\left[\mathbf{1 0}, 4.4\right.$ and 4.7] with an adjunction isomorphism $C R^{n} \Delta^{n} M \rightarrow M$ by Lemma 2.3. The corresponding result for $N \in \hat{A} \Delta$ is obvious.

When $E$ is a spectrum with $K^{n}\left(E ; \hat{\mathbb{Z}}_{2}\right)$ positively torsion-free and $K^{n-1}\left(E ; \hat{\mathbb{Z}}_{2}\right)=$ 0 for some $n$, we now have $K_{C R}^{*}\left(E ; \hat{\mathbb{Z}}_{2}\right) \cong C R^{n}(N)$ in $\hat{\mathcal{A}} \mathcal{R}$ for the torsion-free exact module $N=\Delta^{n} K_{C R}^{*}\left(E ; \hat{\mathbb{Z}}_{2}\right)$ in $\hat{\mathcal{A}} \Delta$, and we have the following existence theorem for such spectra in the stable homotopy category.

Theorem 5.5. For each torsion-free exact $N \in \hat{\mathcal{A}} \Delta$ and $n \in \mathbb{Z}$, there exists a $K / 2_{*}$ local spectrum $\mathcal{E}^{n} N$ with $K_{C R}^{*}\left(\mathcal{E}^{n} N ; \hat{\mathbb{Z}}_{2}\right) \cong C R^{n}(N)$ in $\hat{\mathcal{A C}} \mathcal{R}$. Moreover, $\mathcal{E}^{n} N$ is unique up to (noncanonical) equivalence.

Proof. This follows by Lemma 5.4 and [10, Theorem 5.3].

The spectrum $\mathcal{E}^{n} N$ in the theorem will be endowed with an isomorphism $K_{C R}^{*}\left(\mathcal{E}^{n} N ; \hat{\mathbb{Z}}_{2}\right) \cong C R^{n}(N)$ in $\hat{A} \mathcal{C} \mathcal{R}$. Thus, for an arbitrary spectrum $E$, a map $g: E \rightarrow \mathcal{E}^{n} N$ induces a map $g^{*}: C R^{n}(N) \rightarrow K_{C R}^{*}\left(E ; \hat{\mathbb{Z}}_{2}\right)$ in $\hat{A} \mathcal{C R}$. Each algebraic map of this sort must come from a topological map by:

Theorem 5.6. For a torsion-free exact $N \in \hat{\mathcal{A}} \Delta, n \in \mathbb{Z}$, and an arbitrary spectrum $E$, if $\gamma: C R^{n}(N) \rightarrow K_{C R}^{*}\left(E ; \hat{\mathbb{Z}}_{2}\right)$ is a map in $\hat{A} \mathcal{C} \mathcal{R}$, then there exists a map of spectra $g: E \rightarrow \mathcal{E}^{n} N$ with $g^{*}=\gamma$.

Proof. Let $\tau_{2} E$ denote the 2-torsion part of $E$ given by the homotopy fiber of its localization away from 2. By Pontrjagin duality [10, Theorem 3.1], the map $\gamma$ corresponds to an $A C R$-module map $K_{*}^{C R}\left(\tau_{2} E\right) \rightarrow K_{*}^{C R}\left(\tau_{2} \mathcal{E}^{n} N\right)$ in the sense of [5], where $K_{*}^{C R}\left(\tau_{2} \mathcal{E}^{n} N\right)$ is $C R$-exact with $K_{*}\left(\tau_{2} \mathcal{E}^{n} N\right)$ divisible. This $A C R$-module map prolongs canonically to an $A C R T$-module map $K_{*}^{C R T}\left(\tau_{2} E\right) \rightarrow K_{*}^{C R T}\left(\tau_{2} \mathcal{E}^{n} N\right)$ by $[\mathbf{5}$, Theorem 7.14], and the results of [5, 9.8 and 7.11] now show that this prolonged 
algebraic map must come from a topological map $\tau_{2} E \rightarrow \tau_{2} \mathcal{E}^{n} N$, which gives the desired $g: E \rightarrow \mathcal{E}^{n} N$.

The map $g$ in this theorem is generally not unique (see $[\mathbf{1 0}, 5.4])$.

\section{On the united 2-adic $K$-cohomologies of infinite loop spaces}

In preparation for our work on $K / 2_{*}$-localizations of spaces, we functorially determine the united 2-adic $K$-cohomologies of the needed infinite loop spaces (see Theorem 6.7). We must first introduce:

Definition 6.1 (The 2-adic Adams $\Delta$-modules). By a 2-adic Adams $\Delta$-module $M$, we mean a 2 -adic $\theta \Delta$-module (see Definition 4.3) consisting of stable 2-adic Adams modules $\left\{M_{C}, M_{R}, M_{H}\right\}$ such that the operations $t: M_{C} \cong M_{C}, c: M_{R} \rightarrow M_{C}$, $r: M_{C} \rightarrow M_{R}, c^{\prime}: M_{H} \rightarrow M_{C}, q: M_{C} \rightarrow M_{H}, \theta: M_{C} \rightarrow M_{C}, \theta: M_{R} \rightarrow M_{R}$, and $\theta: M_{H} \rightarrow M_{R}$ are all maps in $\hat{\mathcal{A}}$, where $\psi^{-1}=t$ in $M_{C}$ and $\psi^{-1}=1$ in both $M_{R}$ and $M_{H}$. We let $\hat{\mathcal{M}} \Delta$ denote the abelian category of 2-adic Adams $\Delta$-modules. We say that $M$ is $\theta$-nilpotent when it has $\theta^{i}=0$ for sufficiently large $i$, and we say that $M$ is $\theta$-pro-nilpotent when it is the inverse limit of an inverse system of $\theta$-nilpotent 2 -adic Adams $\Delta$-modules. Thus, $M$ is $\theta$-pro-nilpotent if and only if $M \cong \lim _{i} M / \theta^{i}$ where $M / \theta^{i}$ is the quotient module of $M$ in $\hat{\mathcal{M}} \Delta$ with

$$
\begin{gathered}
\left(M / \theta^{i}\right)_{C}=M_{C} / \theta^{i} M_{C}, \\
\left(M / \theta^{i}\right)_{R}=M_{R} /\left(\theta^{i} M_{R}+\theta^{i} M_{H}+r \theta^{i} M_{C}\right), \\
\left(M / \theta^{i}\right)_{H}=M_{H} / q \theta^{i} M_{C}
\end{gathered}
$$

for $i \geqslant 1$. More simply, $M$ is $\theta$-pro-nilpotent if and only if $\cap_{i} \theta^{i} M_{C}=0$ and $\cap_{i} \theta^{i} M_{R}$ $=0$. It is not hard to show that whenever $M$ is $\theta$-pro-nilpotent, $M$ must be profinite (i.e. $M$ must be the inverse limit of an inverse system of finite 2-adic Adams $\Delta$ modules). For a space $X$, the cohomology

$$
\widetilde{K}_{\Delta}^{-1}\left(X ; \hat{\mathbb{Z}}_{2}\right)=\left\{\widetilde{K}^{-1}\left(X ; \hat{\mathbb{Z}}_{2}\right), \widetilde{K O}^{-1}\left(X ; \hat{\mathbb{Z}}_{2}\right), \bar{S}^{-2} \widetilde{K O}^{-5}\left(X ; \hat{\mathbb{Z}}_{2}\right)\right\}
$$

has a natural 2-adic Adams $\Delta$-module structure by [11, Section 3], and we find:

Lemma 6.2. If $X$ is a connected space with $H^{1}\left(X ; \hat{\mathbb{Z}}_{2}\right)=0$, then the 2-adic Adams $\Delta$-module $\widetilde{K}_{\Delta}^{-1}\left(X ; \hat{\mathbb{Z}}_{2}\right)$ is $\theta$-pro-nilpotent.

Proof. The condition $\cap_{i} \theta^{i} \widetilde{K}^{0}\left(\Sigma X ; \hat{\mathbb{Z}}_{2}\right)=0$ holds by [6, 5.4 and 5.5] since $H^{2}(\Sigma X$; $\left.\hat{\mathbb{Z}}_{2}\right)=0$, and a similar proof shows $\cap_{i} \theta^{i} \widetilde{K O}^{0}\left(\Sigma X ; \hat{\mathbb{Z}}_{2}\right)=0$ since $H^{1}(\Sigma X ; \mathbb{Z} / 2)=0$. This proof uses the fact that the $\lambda$-ideal $\widetilde{K O}^{0} Y$ is $\gamma$-nilpotent for a connected finite CW complex $Y$ by $[\mathbf{1 0}$, Theorem 6.7] and the fact that the real line bundles over $Y$ are classified by $H^{1}(Y ; \mathbb{Z} / 2)$.

Definition 6.3 (The functor $\tilde{F}$ ). We shall construct a functor $\tilde{F}: \hat{\mathcal{A}} \Delta \rightarrow \hat{\mathcal{M}} \Delta$ where $\hat{\mathcal{A}} \Delta$ is the abelian category of stable 2-adic Adams $\Delta$-modules and $\hat{\mathcal{M}} \Delta$ 
is that of 2-adic Adams $\Delta$-modules (see Definitions 5.3 and 6.1). This functor will carry each $N \in \hat{\mathcal{A}} \Delta$ to a universal $\theta$-pro-nilpotent target module $\tilde{F} N \in \hat{\mathcal{M}} \Delta$. For $N \in \hat{\mathcal{A}} \Delta$, we first let $N_{R H} \in \hat{\mathcal{A}}$ denote the pushout of $N_{R} \stackrel{r}{\longleftarrow} N_{C} \stackrel{q}{\longrightarrow} N_{H}$ with a map $\bar{c}: N_{R H} \rightarrow N_{C}$ induced by $c$ and $c^{\prime}$, and with a map $\bar{r}: N_{C} \rightarrow N_{R H}$ induced by $r$ or $q$. We also let $N_{C+} \in \hat{\mathcal{A}}$ denote $N_{C} /(1-t) N_{C}$ and let $N_{C \phi} \in \hat{\mathcal{A}}$ denote $N_{C} /\left(c N_{R}+c^{\prime} N_{H}+2 N_{C}\right)$. We next let

$$
\rho N=\left\{N_{C}, N_{R H} \oplus N_{C \phi}, N_{C+}\right\}
$$

be the stable 2-adic Adams $\Delta$-module with operations given by $t z=t z, c(x, w)=$ $\bar{c} x, r z=(\bar{r} z,[z]), c^{\prime}[z]=(1+t) z$, and $q z=[z]$. We then obtain a stable 2-adic Adams $\Delta$-module

$$
\tilde{F} N=N \times \rho N \times \rho N \times \cdots
$$

with components

$$
\begin{gathered}
\tilde{F}_{C} N=N_{C} \times N_{C} \times N_{C} \times \cdots, \\
\tilde{F}_{R} N=N_{R} \times N_{R H} \times N_{C \phi} \times N_{R H} \times N_{C \phi} \times \cdots, \\
\tilde{F}_{H} N=N_{H} \times N_{C+} \times N_{C+} \times \cdots .
\end{gathered}
$$

We finally define operations $\theta: \tilde{F}_{C} N \rightarrow \tilde{F}_{C} N, \theta: \tilde{F}_{R} N \rightarrow \tilde{F}_{R} N$, and $\theta: \tilde{F}_{H} N \rightarrow$ $\tilde{F}_{R} N$ respectively by the formulae

$$
\begin{aligned}
\theta\left(z_{1}, z_{2}, z_{3}, \ldots\right) & =\left(0, z_{1}, z_{2}, z_{3}, \ldots\right), \\
\theta\left(x_{1}, x_{2}, z_{2}, x_{3}, z_{3}, \ldots\right) & =\left(0,\left[x_{1}\right], 0, x_{2}, 0, x_{3}, 0, \ldots\right), \\
\theta\left(y_{1}, z_{2}, z_{3}, \ldots\right) & =\left(0,\left[y_{1}\right], 0, \bar{r} z_{2}, 0, \bar{r} z_{3}, 0, \ldots\right) .
\end{aligned}
$$

This gives a natural 2-adic Adams $\Delta$-module $\tilde{F} N$ and hence a functor $\tilde{F}: \hat{\mathcal{A}} \Delta \rightarrow$ $\hat{\mathcal{M}} \Delta$. We let $\iota: N \rightarrow \tilde{F} N$ be the map in $\hat{\mathcal{A}} \Delta$ with $\iota_{C}(z)=(z, 0,0, \ldots), \iota_{R}(x)=$ $(x, 0,0, \ldots)$, and $\iota_{H}(y)=(y, 0,0, \ldots)$, and we show:

Theorem 6.4. For a stable 2-adic Adams $\Delta$-module $N \in \hat{\mathcal{A}} \Delta$, the 2-adic Adams $\Delta$-module $\tilde{F} N \in \hat{\mathcal{M}} \Delta$ is $\theta$-pro-nilpotent and the map $\iota: N \rightarrow \tilde{F} N$ has the universal property that, for each $\theta$-pro-nilpotent $M \in \hat{\mathcal{M}} \Delta$ and map $f: N \rightarrow M$ in $\hat{\mathcal{A}} \Delta$, there exists a unique map $\bar{f}: \tilde{F} N \rightarrow M$ in $\hat{\mathcal{M}} \Delta$ with $\bar{f} \iota=f$.

Proof. $\tilde{F} N$ is $\theta$-pro-nilpotent since it is the inverse limit of its quotient modules

$$
\tilde{F} N / \theta^{i+1} \cong N \times \rho N \times \cdots \times \rho N .
$$

For $i \geqslant 1$, we define a map $f^{(i)}: \rho N \rightarrow M$ in $\hat{\mathcal{A}} \Delta$ by

$$
\begin{gathered}
f_{C}^{(i)}=\theta^{i} f_{C}: N_{C} \longrightarrow M_{C}, \\
f_{R}^{(i)}=\left(\theta^{i} f_{R}, \theta^{i} f_{H}\right)+\bar{\phi} \theta^{i-1} f_{C}: N_{R H} \oplus N_{C \phi} \longrightarrow M_{R}, \\
f_{H}^{(i)}=q \theta^{i} f_{C}: N_{C+} \longrightarrow M_{H} .
\end{gathered}
$$

We then define $\bar{f}: \tilde{F} N \rightarrow M$ as the inverse limit of the maps

$$
f+f^{(1)}+\cdots+f^{(i)}: N \times \rho N \times \cdots \times \rho N \longrightarrow M / \theta^{i+1}
$$

in $\hat{\mathcal{M}} \Delta$, and we check that $\bar{f} \iota=f$. The uniqueness condition for $\bar{f}$ follows since the 
2-adic Adams $\Delta$-modules $\tilde{F} N / \theta^{i+1}=N \times \rho N \times \cdots \times \rho N$ are generated by $\iota N$.

To show the robustness (see Definition 4.7) of $\tilde{F} N$ for suitable $N$, we need:

Definition 6.5 (The functor $\bar{\rho}: \hat{\mathcal{A}} \Delta \rightarrow \hat{\mathcal{A}} \Delta$ ). For $N \in \hat{\mathcal{A}} \Delta$, we let $\bar{\rho} N=\left\{N_{C}\right.$, $\left.N_{R H}, N_{C+}\right\}$ be the stable 2-adic Adams $\Delta$-module with operations given by $t z=$ $t z, \quad c x=\bar{c} x, \quad r z=\bar{r} z, \quad c^{\prime}[z]=(1+t) z$, and $q z=[z]$. Thus, $\bar{\rho} N$ is the quotient of $\rho N=\left\{N_{C}, N_{R H} \oplus N_{C \phi}, N_{C+}\right\}$ by $N_{C \phi}$. If $N$ is torsion-free and exact, then $\bar{\rho} N$ is also torsion-free and exact by Lemma 4.2 since it is isomorphic to the module $\left\{N_{C}, N_{R}+N_{H}, N_{R} \cap N_{H}\right\}$ with $c$ and $c^{\prime}$ treated as inclusions.

Lemma 6.6. If $N \in \hat{\mathcal{A}} \Delta$ is torsion-free and exact, then $\tilde{F} N \in \hat{\mathcal{M}} \Delta$ is robust.

Proof. We check that $\bar{\phi}: \tilde{F}_{C} N \rightarrow \tilde{F}_{R} N$ is given by

$$
\bar{\phi}\left(z_{1}, z_{2}, z_{3}, \ldots\right)=\left(0,0,\left[z_{1}\right], 0,\left[z_{2}\right], 0, \ldots\right)
$$

for $z_{i} \in N_{C}$ and $\left[z_{i}\right] \in N_{C \phi}$. Thus, $\operatorname{ker} \bar{\phi}=c \tilde{F}_{R} N+c^{\prime} \tilde{F}_{H} N+2 \tilde{F}_{C} N$ and $\tilde{F} N / \bar{\phi} \cong$ $N \times \bar{\rho} N \times \bar{\rho} N \times \cdots$. Hence, $\tilde{F} N / \bar{\phi}$ is torsion-free and exact by Definition 6.5 as required.

Our main result in this section is:

Theorem 6.7. If $E$ is a 0-connected spectrum with $H^{1}\left(E ; \hat{\mathbb{Z}}_{2}\right)=0=H^{2}\left(E ; \hat{\mathbb{Z}}_{2}\right)$, with $K^{0}\left(E ; \hat{\mathbb{Z}}_{2}\right)=0$, and with $K^{-1}\left(E ; \hat{\mathbb{Z}}_{2}\right)$ positively torsion-free (5.3), then there is a natural isomorphism $\hat{L} \tilde{F} K_{\Delta}^{-1}\left(E ; \hat{\mathbb{Z}}_{2}\right) \cong K_{C R}^{*}\left(\Omega^{\infty} E ; \hat{\mathbb{Z}}_{2}\right)$.

Proof. Since $\widetilde{K}_{\Delta}^{-1}\left(\Omega^{\infty} E ; \hat{\mathbb{Z}}_{2}\right)$ is $\theta$-pro-nilpotent by Lemma 6.2 , the infinite suspension map $\sigma: K_{\Delta}^{-1}\left(E ; \hat{\mathbb{Z}}_{2}\right) \rightarrow \widetilde{K}_{\Delta}^{-1}\left(\Omega^{\infty} E ; \hat{\mathbb{Z}}_{2}\right)$ induces a map $\bar{\sigma}: \tilde{F} K_{\Delta}^{-1}\left(E ; \hat{\mathbb{Z}}_{2}\right) \rightarrow$ $\widetilde{K}_{\Delta}^{-1}\left(\Omega^{\infty} E ; \hat{\mathbb{Z}}_{2}\right)$ in $\hat{\mathcal{M}} \Delta$, where $\tilde{F} K_{\Delta}^{-1}\left(E ; \hat{\mathbb{Z}}_{2}\right)$ is robust by Lemmas 5.4 and 6.6. Thus $\bar{\sigma}$ induces an isomorphism $\hat{L} \tilde{F} K_{\Delta}^{-1}\left(E ; \hat{\mathbb{Z}}_{2}\right) \cong K_{C R}^{*}\left(\Omega^{\infty} E ; \hat{\mathbb{Z}}_{2}\right)$ by Theorem 4.9, since it induces an isomorphism of the complex components by [6, Theorem 8.3].

\section{Strong 2-adic Adams $\Delta$-modules}

Our main results on $K / 2_{*}$-localizations in Section 8 will involve a space $X$ with $K_{C R}^{*}\left(X ; \hat{\mathbb{Z}}_{2}\right) \cong \hat{L} M$ for a 2 -adic Adams $\Delta$-module $M$ that is strong in the sense that it is robust, $\psi^{3}$-splittable, and regular. In this section, we provide the required algebraic definitions and explanations of these notions. We first recall:

Definition 7.1 (The robust modules). We say that a 2-adic Adams $\Delta$-module $M$ is robust when it is robust in the sense of Definition 4.7, ignoring stable Adams operations. When $M$ is robust, the underlying 2-adic $\Delta$-module $M / \bar{\phi}$ satisfies the conditions of Lemma 4.2 and may be factored as a (possibly infinite) product of 
monogenic free 2 -adic $\Delta$-modules

$$
\begin{gathered}
F^{C}(z)=\left\{\hat{\mathbb{Z}}_{2} \oplus t \hat{\mathbb{Z}}_{2}, \hat{\mathbb{Z}}_{2}, \hat{\mathbb{Z}}_{2}\right\}=\{\langle z\rangle \oplus\langle t z\rangle,\langle r z\rangle,\langle q z\rangle\}, \\
F^{R}(x)=\left\{\hat{\mathbb{Z}}_{2}, \hat{\mathbb{Z}}_{2}, \hat{\mathbb{Z}}_{2}\right\}=\{\langle c x\rangle,\langle x\rangle,\langle q c x\rangle\}, \\
F^{H}(y)=\left\{\hat{\mathbb{Z}}_{2}, \hat{\mathbb{Z}}_{2}, \hat{\mathbb{Z}}_{2}\right\}=\left\{\left\langle c^{\prime} y\right\rangle,\left\langle r c^{\prime} y\right\rangle,\langle y\rangle\right\}
\end{gathered}
$$

by an argument using the factorization of positively torsion-free groups in Definition 5.3. We let $\operatorname{gen}_{C} M$, gen ${ }_{R} M$, and $\operatorname{gen}_{H} M$ respectively denote the number of complex, real, and quaternionic monogenic free factors of $M / \bar{\phi}$. These numbers do not depend on the factorization since they equal the dimensions of the respective $\mathbb{Z} / 2$-vector spaces $\left(M_{C \phi}\right)^{\#},\left(M_{R} /\left(\bar{\phi} M_{C}+r M_{C}\right)\right)^{\#}$, and $\left(M_{H} / q M_{C}\right)^{\#}$, where $(-)^{\#}$ is the Pontrjagin duality functor from 2-profinite abelian groups to discrete 2 -torsion abelian groups. Using the factorization of $M / \bar{\phi}$, we find that

$$
\text { gen } M_{C}=2 \operatorname{gen}_{C} M+\operatorname{gen}_{R} M+\operatorname{gen}_{H} M
$$

where gen $M_{C}$ denotes the number of $\hat{\mathbb{Z}}_{2}$ factors in the 2-profinite abelian group $M_{C}$.

Definition 7.2 (The $\psi^{3}$-splittable modules). For a 2 -adic Adams $\Delta$-module $M \in$ $\hat{\mathcal{M}} \Delta$, we consider the stable 2-adic Adams $\Delta$-module $\bar{M}=M / \bar{\phi} \in \hat{\mathcal{A}} \Delta$, and we say that $M$ is $\psi^{3}$-splittable when the quotient map $M \rightarrow \bar{M}$ has a right inverse $s: \bar{M} \rightarrow$ $M$ in $\hat{\mathcal{A}} \Delta$. We call such a map $s$ a $\psi^{3}$-splitting of $M$, and we note that it corresponds to a left inverse $s^{\prime}: M_{R} / r M_{C} \rightarrow \bar{\phi} M_{C}$ of the canonical map $\bar{\phi} M_{C} \rightarrow M_{R} / r M_{C}$ in the category $\hat{\mathcal{A}}$ of stable 2 -adic Adams modules, or equivalently in the category of profinite $\mathbb{Z} / 2$-modules with automorphisms $\psi^{3}$. We deduce that $M$ is automatically $\psi^{3}$-splittable in some important cases:

Lemma 7.3. If $M$ is a robust 2-adic Adams $\Delta$-module with gen $_{C} M=0$ or gen $_{R} M$ $=0$, then $M$ is $\psi^{3}$-splittable.

Proof. Since $M_{C}$ is positively torsion-free, the map $\mathrm{cr}=1+t: M_{C+} \rightarrow M_{C}$ is monic, and hence $c: r M_{C} \rightarrow M_{C}$ is also monic. Thus, $\bar{\phi} M_{C} \cap r M_{C}=0$ and there is a short exact sequence

$$
0 \longrightarrow \bar{\phi} M_{C} \longrightarrow M_{R} / r M_{C} \longrightarrow M_{R} /\left(\bar{\phi} M_{C}+r M_{C}\right) \longrightarrow 0
$$

in $\hat{\mathcal{A}}$. Since gen ge $_{C} M=0$ or gen ${ }_{R} M=0$, this has $\bar{\phi} M_{C}=0$ or $M_{R} /\left(\bar{\phi} M_{C}+r M_{C}\right)=$ 0 , and hence the map $\bar{\phi} M_{C} \rightarrow M_{R} / r M_{C}$ has an obvious left inverse in $\hat{\mathcal{A}}$.

We shall use the $\psi^{3}$-splittability condition to give:

Definition 7.4 (The $\theta$-resolutions of modules). Let $M \in \hat{\mathcal{M}} \Delta$ be a 2-adic Adams $\Delta$-module that is $\theta$-pro-nilpotent, robust, and $\psi^{3}$-splittable. These conditions will hold when $M$ is strong (see Definition 7.11). For a $\psi^{3}$-splitting $s: \bar{M} \rightarrow M$ in $\hat{\mathcal{A}} \Delta$, we shall construct an associated $\theta$-resolution

$$
0 \longrightarrow \tilde{F} \bar{\rho} \bar{M} \stackrel{\bar{d}}{\longrightarrow} \tilde{F} \bar{M} \stackrel{\bar{s}}{\longrightarrow} M \longrightarrow 0
$$

of $M$ in $\hat{\mathcal{M}} \Delta$, with $\bar{\rho} \bar{M}=\left\{\bar{M}_{C}, \bar{M}_{R H}, \bar{M}_{C+}\right\}$ as in Definition 6.5 , where $\bar{s}: \tilde{F} \bar{M} \rightarrow$ 
$M$ is induced by $s$ via Theorem 6.4. To specify $\bar{d}$, we use the commutative square

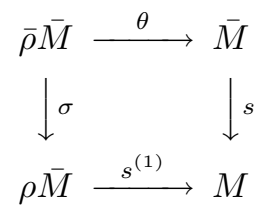

in $\hat{\mathcal{A}} \Delta$ with $\rho \bar{M}=\left\{\bar{M}_{C}, \bar{M}_{R H} \oplus \bar{M}_{C \phi}, \bar{M}_{C+}\right\}$ as in Definition 6.3, where $s^{(1)}$ is given by the proof of Theorem 6.4 , where $\theta=\{\theta,(\theta, \theta), q \theta\}$, and where $\sigma=\left\{1,\left(1, \theta_{\phi}\right), 1\right\}$, using the map $\theta_{\phi}: \bar{M}_{R H} \rightarrow \bar{M}_{C \phi}=M_{C \phi}$ given by the composition of the sequence

$$
\bar{M}_{R H} \stackrel{s}{\longrightarrow} M_{R H} \stackrel{(\theta, \theta)}{\longrightarrow} M_{R} \cong \bar{M}_{R} \oplus M_{C \phi} \stackrel{\text { proj }}{\longrightarrow} M_{C \phi}
$$

in which the isomorphism is the inverse of $(s, \bar{\phi}): \bar{M}_{R} \oplus M_{C \phi} \cong M_{R}$. The commutative square now gives a map

$$
d=(\theta,-\sigma, 0,0, \ldots): \bar{\rho} \bar{M} \rightarrow \tilde{F} \bar{M}
$$

in $\hat{\mathcal{A}} \Delta$ with $\bar{s} d=0$, and this induces the required map $\bar{d}: \tilde{F} \bar{\rho} \bar{M} \rightarrow \tilde{F} \bar{M}$ in $\hat{\mathcal{M}} \Delta$ with $\bar{s} \bar{d}=0$.

Lemma 7.5. If $M \in \hat{\mathcal{M}} \Delta$ is $\theta$-pro-nilpotent and robust with a $\psi^{3}$-splitting $s: \bar{M} \rightarrow$ $M$, then the $\theta$-resolution $0 \rightarrow \tilde{F} \bar{\rho} \bar{M} \stackrel{\bar{d}}{\rightarrow} \tilde{F} \bar{M} \stackrel{\bar{s}}{\rightarrow} M \rightarrow 0$ is exact in $\hat{\mathcal{M}} \Delta$.

Proof. We easily check that $0 \rightarrow \bar{\phi}(\tilde{F} \bar{\rho} \bar{M})_{C} \rightarrow \bar{\phi}(\tilde{F} \bar{M})_{C} \rightarrow \bar{\phi} M_{C} \rightarrow 0$ is exact and that $\bar{s} / \bar{\phi}: \tilde{F} \bar{M} / \bar{\phi} \rightarrow M / \bar{\phi}$ is onto. Hence, it suffices to show that the map $\tilde{F} \bar{\rho} \bar{M} / \bar{\phi} \rightarrow$ $\operatorname{ker}(\bar{s} / \bar{\phi})$ is an isomorphism. This follows by [10, Lemma 4.8] since the map $(\tilde{F} \bar{\rho} \bar{M} / \bar{\phi})_{C} \rightarrow \operatorname{ker}(\bar{s} / \bar{\phi})_{C}$ is clearly an isomorphism and since the 2 -adic $\Delta$-modules $\tilde{F} \bar{\rho} \bar{M} / \bar{\phi}$ and $\operatorname{ker}(\bar{s} / \bar{\phi})$ are exact by Lemma 6.6 and by the short exact sequence rule of $[\mathbf{1 0}, 4.5]$.

To formulate our regularity condition for $M$, we use:

Definition 7.6 (The 2-adic Adams modules). These are the unstable versions of the stable 2-adic Adams modules and were previously discussed in [8, 2.8]. By a finite 2-adic Adams module A, we mean a finite abelian 2-group with endomorphisms $\psi^{k}: A \rightarrow A$ for $k \in \mathbb{Z}$ such that:

(i) $\psi^{1}=1$ and $\psi^{j} \psi^{k}=\psi^{j k}$ for $j, k \in \mathbb{Z}$;

(ii) when $n$ is sufficiently large, the condition $j \equiv k \bmod 2^{n}$ implies $\psi^{j}=\psi^{k}$.

By a 2-adic Adams module $A$, we mean the topological inverse limit of an inverse system of finite 2-adic Adams modules. Such an $A$ has an underlying 2-profinite abelian group with continuous endomorphisms $\psi^{k}: A \rightarrow A$ for $k \in \mathbb{Z}$ (and in fact for $\left.k \in \hat{\mathbb{Z}}_{2}\right)$. For a space $X$, the cohomology $K^{1}\left(X ; \hat{\mathbb{Z}}_{2}\right)$ is a 2-adic Adams module with the usual Adams operations $\psi^{k}$ for $k \in \mathbb{Z}$ as in [6, Example 5.2]. We note that the operations $\psi^{2}$ and $\psi^{k}$, for $k$ odd, in $K^{1}\left(X ; \hat{\mathbb{Z}}_{2}\right)$ correspond via Bott periodicity to $\theta$ and to $k^{-1} \psi^{k}$ in $K^{-1}\left(X ; \hat{\mathbb{Z}}_{2}\right)$. In general, for a $\theta$-pro-nilpotent 2-adic Adams $\Delta$-module $M$, we obtain a 2-adic Adams module $M^{C}$ having the same group as $M_{C}$ but having $\psi^{0}=0$ and having $\psi^{k 2^{i}}$ equal to $k^{-1} \psi^{k} \theta^{i}$ on $M_{C}$ for $k$ odd and $i \geqslant 0$. 
Definition 7.7 (The linear and strictly nonlinear modules). As in [8, Section 4] and [7, Section 2], a 2-adic Adams module $H$ is called linear when it has $\psi^{k}=k$ for all $k \in \mathbb{Z}$, and $H$ is called quasilinear when $2 H \subset \psi^{2} H$. Each 2-adic Adams module $A$ has a largest linear quotient module

$$
\operatorname{Lin} A=A /\left(\left(\psi^{2}-2\right) A+\left(\psi^{-1}+1\right) A+\left(\psi^{3}-3\right) A\right)
$$

and also has a largest quasilinear submodule $A_{q l} \subset A$ by Lemma 13.1 below. A 2adic Adams module $A$ is called strictly nonlinear when $A_{q l}=0$. This implies that $A$ is torsion-free with $\cap_{i}\left(\psi^{2}\right)^{i} A=0$, and $A$ will be strictly nonlinear by Remark 13.2 and $[\mathbf{7}, 2.5]$ whenever it is torsion-free with $\left(\psi^{2}\right)^{i} A \subset 2^{i+1} A$ for some $i \geqslant 1$.

Definition 7.8 (The regular modules). As in $[8,4.4]$, we say that a 2-adic Adams module $A$ is regular when the kernel of $A \rightarrow \operatorname{Lin} A$ is strictly nonlinear. This implies that $\cap_{i}\left(\psi^{2}\right)^{i} A=0$, and $A$ will be regular whenever it is an extension of a strictly nonlinear submodule by a linear quotient module. We also say that a 2-adic Adams $\Delta$-module $M$ is regular when it is $\theta$-pro-nilpotent with $M^{C}$ regular as a 2-adic Adams module. For a connected space $X$ with $H^{1}\left(X ; \hat{\mathbb{Z}}_{2}\right)=0$, the 2 -adic Adams $\Delta$ module $\widetilde{K}_{\Delta}^{-1}\left(X ; \hat{\mathbb{Z}}_{2}\right)$ is always $\theta$-pro-nilpotent by Lemma 6.2 , and hence $\widetilde{K}_{\Delta}^{-1}\left(X ; \hat{\mathbb{Z}}_{2}\right)$ is regular if and only if $\widetilde{K}^{1}\left(X ; \hat{\mathbb{Z}}_{2}\right)$ is regular as a 2 -adic Adams module. The following two lemmas will often guarantee regularity for our modules.

Lemma 7.9. Let $X$ be a connected space with $H^{1}\left(X ; \hat{\mathbb{Z}}_{2}\right)=0$, with $H^{m}\left(X ; \hat{\mathbb{Z}}_{2}\right)=0$ for sufficiently large $m$, and with $\widetilde{K}^{1}\left(X ; \hat{\mathbb{Z}}_{2}\right)$ torsion-free. Then $\widetilde{K}^{1}\left(X ; \hat{\mathbb{Z}}_{2}\right)$ is regular with $\psi^{2}: \widetilde{K}^{1}\left(X ; \hat{\mathbb{Z}}_{2}\right) \rightarrow \widetilde{K}^{1}\left(X ; \hat{\mathbb{Z}}_{2}\right)$ monic, and hence $\widetilde{K}_{\Delta}^{-1}\left(X ; \hat{\mathbb{Z}}_{2}\right)$ is regular with $\theta: \widetilde{K}^{-1}\left(X ; \hat{\mathbb{Z}}_{2}\right) \rightarrow \widetilde{K}^{-1}\left(X ; \hat{\mathbb{Z}}_{2}\right)$ monic.

Lemma 7.10. For a regular 2-adic Adams module A, each submodule is regular, and each torsion-free quotient module is regular when $A$ is finitely generated over $\hat{\mathbb{Z}}_{2}$.

The proofs are in Section 13. Combining the preceding definitions, we finally introduce:

Definition 7.11 (The strong modules). We say that a 2 -adic Adams $\Delta$-module $M \in \hat{\mathcal{M}} \Delta$ is strong when:

(i) $M$ is robust;

(ii) $M$ is $\psi^{3}$-splittable;

(iii) $M$ is regular.

Such an $M$ is automatically $\theta$-pro-nilpotent (and hence profinite) since it is regular.

\section{On the $K / 2_{*}$-localizations of our spaces}

We recall that the $K / 2_{*}$-localizations of spaces or spectra are the same as the $K^{*}\left(-; \hat{\mathbb{Z}}_{2}\right)$-localizations since the $K / 2_{*}$-equivalences are the same as the $K^{*}\left(-; \hat{\mathbb{Z}}_{2}\right)$ equivalences. In this section, we give our main result (Theorem 8.6) on the $K / 2_{*^{-}}$ localization of a connected space $X$ with $K_{C R}^{*}\left(X ; \hat{\mathbb{Z}}_{2}\right) \cong \hat{L} M$ for a strong 2-adic Adams $\Delta$-module $M$. We first consider: 
Definition 8.1 (Building blocks for $K / 2_{*}$-localizations). For a torsion-free exact stable 2-adic Adams $\Delta$-module $N \in \hat{\mathcal{A}} \Delta$, we let $\mathcal{E} N$ denote the $K / 2_{*}$-local spectrum $\mathcal{E}^{-1} N$ of Theorem 5.5 with an isomorphism $K_{C R}^{*}\left(\mathcal{E} N ; \hat{\mathbb{Z}}_{2}\right) \cong C R^{-1} N$ in the category $\hat{\mathcal{A} C \mathcal{R}}$ of stable 2-adic Adams $C R$-modules. As in [8,3.5], we let $\tilde{\mathcal{E}} N \rightarrow \mathcal{E} N \rightarrow \bar{P}^{2} \mathcal{E} N$ denote the Postnikov fiber sequence of spectra with $\pi_{i} \tilde{\mathcal{E}} N \cong \pi_{i} \mathcal{E} N$ for $i>2$, with $\pi_{i} \tilde{\mathcal{E}} N=0$ for $i<2$, and with $\pi_{2} \tilde{\mathcal{E}} N \cong \hat{t}_{2} \pi_{2} \mathcal{E} N$, where $\hat{t}_{2} \pi_{2} \tilde{\mathcal{E}} N \subset \pi_{2} \mathcal{E} N$ denotes the Ext-2-completion of the torsion subgroup of $\pi_{2} \mathcal{E} N$. We now obtain a simplyconnected infinite loop space $\Omega^{\infty} \tilde{\mathcal{E}} N$ which is $K / 2_{*}$-local by [8, Theorem 3.8]. These $\Omega^{\infty} \tilde{\mathcal{E}} N$, with their companions $\Omega^{\infty} \tilde{\mathcal{E}} \bar{\rho} N$, will serve as our building blocks for $K / 2_{*^{-}}$ localizations of spaces, where $\bar{\rho} N$ denotes the torsion-free exact stable 2-adic Adams $\Delta$-module $\bar{\rho} N=\left\{N_{C}, N_{R}+N_{H}, N_{R} \cap N_{H}\right\}$ of Definition 6.5.

Definition 8.2 (Strict homomorphisms and isomorphisms). For a 2-adic Adams $\Delta$-module $M \in \hat{\mathcal{M}} \Delta$ and a connected space $X$, a strict homomorphism (resp. strict isomorphism) $\hat{L} M \rightarrow K_{C R}^{*}\left(X ; \hat{\mathbb{Z}}_{2}\right)$ is a homomorphism (resp. isomorphism) of special 2-adic $\phi C R$-algebras induced by a map $M \rightarrow \widetilde{K}_{\Delta}^{-1}\left(X ; \hat{\mathbb{Z}}_{2}\right)$ of 2-adic Adams $\Delta$-modules. For instance, there is a strict isomorphism

$$
\hat{L} \tilde{F} N \cong K_{C R}^{*}\left(\Omega^{\infty} \tilde{\mathcal{E}} N ; \hat{\mathbb{Z}}_{2}\right)
$$

for each torsion-free exact stable 2 -adic Adams $\Delta$-module $N \in \hat{\mathcal{A}} \Delta$ by Theorem 6.7, and we have:

Lemma 8.3. For a torsion-free exact module $N \in \hat{\mathcal{A}} \Delta$ and a connected space $X$ with $H^{1}\left(X ; \hat{\mathbb{Z}}_{2}\right)=0=H^{2}\left(X ; \hat{\mathbb{Z}}_{2}\right)$, each strict homomorphism $\hat{L} \tilde{F} N \rightarrow K_{C R}^{*}\left(X ; \hat{\mathbb{Z}}_{2}\right)$ is induced by a (possibly non-unique) map $X \rightarrow \Omega^{\infty} \tilde{\mathcal{E}} N$.

Proof. A strict homomorphism $\hat{L} \tilde{F} N \rightarrow K_{C R}^{*}\left(X ; \hat{\mathbb{Z}}_{2}\right)$ corresponds successively to: a map $\tilde{F} N \rightarrow \widetilde{K}_{\Delta}^{-1}\left(X ; \hat{\mathbb{Z}}_{2}\right)$ in $\hat{\mathcal{M}} \Delta$, a map $N \rightarrow \widetilde{K}_{\Delta}^{-1}\left(X ; \hat{\mathbb{Z}}_{2}\right)$ in $\hat{\mathcal{A}} \Delta$, and a map $C R^{-1} N \rightarrow K_{C R}^{*}\left(\Sigma^{\infty} X ; \hat{\mathbb{Z}}_{2}\right)$ in $\hat{\mathcal{A}} \mathcal{C}$. By Theorem 5.6 , this last map is induced by a map $\Sigma^{\infty} X \rightarrow \mathcal{E} N$, which lifts uniquely to a map $\Sigma^{\infty} X \rightarrow \tilde{\mathcal{E}} N$, and we can easily check that the adjoint map $X \rightarrow \Omega^{\infty} \tilde{\mathcal{E}} N$ induces the original strict homomorphism.

Definition 8.4 (The key construction). For a strong 2-adic Adams $\Delta$-module $M \in$ $\hat{\mathcal{M}} \Delta$, we may take a $\theta$-resolution (see Definition 7.4)

$$
0 \longrightarrow \tilde{F} \bar{\rho} \bar{M} \stackrel{\bar{d}}{\longrightarrow} \tilde{F} \bar{M} \stackrel{\bar{s}}{\longrightarrow} M \longrightarrow 0
$$

using the torsion-free exact module $\bar{M}=M / \bar{\phi} \in \hat{\mathcal{A}} \Delta$. We may then apply Lemma 8.3 to give a map $f: \Omega^{\infty} \tilde{\mathcal{E}} \bar{M} \rightarrow \Omega^{\infty} \tilde{\mathcal{E}} \bar{\rho} \bar{M}$ inducing the $K_{C R}^{*}\left(-; \hat{\mathbb{Z}}_{2}\right)$-homomorphism $f^{*}=\hat{L} \bar{d}: \hat{L} \tilde{F} \bar{\rho} \bar{M} \rightarrow \hat{L} \tilde{F} M$. Any such $f$ will be called a companion map of $M$, and its homotopy fiber Fib $f$ will be $K / 2_{*}$-local since $\Omega^{\infty} \tilde{\mathcal{E}} M$ and $\Omega^{\infty} \tilde{\mathcal{E}} \bar{\rho} M$ are. As in $[\mathbf{8}, 4.6]$ and Definition 8.1, we let

$$
\widetilde{\mathrm{Fib}} f \longrightarrow \text { Fib } f \longrightarrow \bar{P}^{2} \text { Fib } f
$$

denote the Postnikov fiber sequence with $\pi_{i} \widetilde{\operatorname{Fib}} f \cong \pi_{i}$ Fib $f$ for $i>2$, with $\pi_{i} \widetilde{\text { Fib }} f=$ 0 for $i<2$, and with $\pi_{i} \widetilde{\mathrm{Fib}} f \cong \hat{t}_{2} \pi_{2} \widetilde{\mathrm{Fib}} f$. We note that $\bar{P}^{2}$ Fib $f$ is an infinite loop 
space which is $K / 2_{*}$-local by [8, Theorem 3.8], and we conclude that $\widetilde{\text { Fib }} f$ is also $K / 2_{*}$-local. Moreover, we have $K_{C R}^{*}\left(\widetilde{\mathrm{Fib}} f ; \hat{\mathbb{Z}}_{2}\right) \cong \hat{L} M$ by:

Theorem 8.5. For a strong 2-adic Adams $\Delta$-module $M \in \hat{\mathcal{M}} \Delta$ and any companion map $f: \Omega^{\infty} \tilde{\mathcal{E}} \bar{M} \rightarrow \Omega^{\infty} \tilde{\mathcal{E}} \bar{\rho} \bar{M}$, there is a strict isomorphism $\hat{L} M \cong K_{C R}^{*}\left(\widetilde{\operatorname{Fib} f} ; \hat{\mathbb{Z}}_{2}\right)$.

Thus, $\hat{L} M$ is topologically realizable for each strong $M \in \hat{\mathcal{M}} \Delta$. This theorem will be proved in Section 14 and leads immediately to our main result on $K / 2_{*^{-}}$ localizations of spaces.

Theorem 8.6. If $X$ is a connected space with a strict isomorphism $\hat{L} M \cong K_{C R}^{*}(X$; $\left.\hat{\mathbb{Z}}_{2}\right)$ for a strong 2-adic Adams $\Delta$-module $M \in \hat{\mathcal{M}} \Delta$, then there is an equivalence $X_{K / 2} \simeq \widetilde{\operatorname{Fib} f}$ for some companion map $f: \Omega^{\infty} \tilde{\mathcal{E}} \bar{M} \rightarrow \Omega^{\infty} \tilde{\mathcal{E}} \bar{\rho} \bar{M}$ of $M$, where the equivalence induces the canonical isomorphism $K_{C R}^{*}\left(\widetilde{\mathrm{Fib}} ; \hat{\mathbb{Z}}_{2}\right) \cong \hat{L} M \cong K_{C R}^{*}(X$; $\left.\hat{\mathbb{Z}}_{2}\right)$. Moreover, $H^{1}\left(X ; \hat{\mathbb{Z}}_{2}\right)=0=H^{2}\left(X ; \hat{\mathbb{Z}}_{2}\right)$.

Proof. The last statement follows by $[6,5.4]$. For the first, we take a $\theta$-resolution $0 \rightarrow \tilde{F} \bar{\rho} \bar{M} \stackrel{\bar{d}}{\rightarrow} \tilde{F} \bar{M} \stackrel{\bar{s}}{\rightarrow} M \rightarrow 0$ of $M$ and apply Lemma 8.3 to give a map $h: X \rightarrow$ $\Omega^{\infty} \tilde{\mathcal{E}} \bar{M}$ with $h^{*}=\hat{L} \bar{s}: \hat{L} \tilde{F} \bar{M} \rightarrow \hat{L} M$. We then apply Lemma 8.3 again to give a map $k:$ Cof $h \rightarrow \Omega^{\infty} \tilde{\mathcal{E}} \bar{\rho} \bar{M}$ with

$$
k^{*}=\hat{L} \bar{d}: \hat{L} \tilde{F} \bar{\rho} \bar{M} \longrightarrow K_{C R}^{*}\left(\operatorname{Cof} h ; \hat{\mathbb{Z}}_{2}\right) \subset \hat{L} \tilde{F} \bar{M} .
$$

Composing $k$ with the cofiber map, we obtain a companion map $f: \Omega^{\infty} \tilde{\mathcal{E}} \bar{M} \rightarrow$ $\Omega^{\infty} \tilde{\mathcal{E}} \bar{\rho} \bar{M}$ of $M$ such that $h$ lifts to a map $u: X \rightarrow \widetilde{\mathrm{Fib}} f$ which is a $K / 2_{*}$-equivalence by Theorem 8.5. Since $\widetilde{\mathrm{Fib}} f$ is $K / 2_{*}$-local, this gives the desired equivalence $X_{K / 2} \simeq$ $\widetilde{\operatorname{Fib} f}$.

In this theorem, $M$ is uniquely determined by the space $X$ since there is a canonical isomorphism $M \cong \hat{Q} K_{\Delta}^{-1}\left(X ; \hat{\mathbb{Z}}_{2}\right)$ in $\hat{\mathcal{M}} \Delta$ by Remark 4.10 and [11, Section 3].

\section{On the $v_{1}$-periodic homotopy groups of our spaces}

The $p$-primary $v_{1}$-periodic homotopy groups $v_{1}^{-1} \pi_{*} X$ of a space $X$ at a prime $p$ were defined by Davis and Mahowald [15] and have been studied extensively (see $[\mathbf{1 3}]$ ). In this section, we apply the preceding result (Theorem 8.6) on the $K / 2_{*}$-localizations of our spaces to approach $v_{1}$-periodic homotopy groups at $p=2$ using:

Definition 9.1 (The functor $\Phi_{1}$ ). As in $[\mathbf{4}],[\mathbf{9}],[\mathbf{1 6}]$, and [18], there is a $v_{1}$ stabilization functor $\Phi_{1}$ from the homotopy category of spaces to that of spectra such that:

(i) for a space $X$, there is a natural isomorphism $v_{1}^{-1} \pi_{*} X \cong \pi_{*} \tau_{2} \Phi_{1} X$ where $\tau_{2} \Phi_{1} X$ is the 2 -torsion part of $\Phi_{1} X$ (given by the fiber of its localization away from 2);

(ii) $\Phi_{1} X$ is $K / 2_{*}$-local for each space $X$; 
(iii) for a spectrum $E$, there is a natural equivalence $\Phi_{1}\left(\Omega^{\infty} E\right) \simeq E_{K / 2}$;

(iv) $\Phi_{1}$ preserves fiber squares.

Various other properties of $\Phi_{1}$ are described in [10, Section 2], and the isomorphism $v_{1}^{-1} \pi_{*} X \cong \pi_{*} \tau_{2} \Phi_{1} X$ may be applied as in [10, Theorem 3.2] to show:

Theorem 9.2. For a space $X$, there is a natural long exact sequence

$$
\begin{gathered}
\cdots \longrightarrow K O^{n-3}\left(\Phi_{1} X ; \hat{\mathbb{Z}}_{2}\right) \stackrel{\psi^{3}-9}{\longrightarrow} K O^{n-3}\left(\Phi_{1} X ; \hat{\mathbb{Z}}_{2}\right) \longrightarrow\left(v_{1}^{-1} \pi_{n} X\right)^{\#} \\
\longrightarrow K O^{n-2}\left(\Phi_{1} X ; \hat{\mathbb{Z}}_{2}\right) \stackrel{\psi^{3}-9}{\longrightarrow} K O^{n-2}\left(\Phi_{1} X ; \hat{\mathbb{Z}}_{2}\right) \longrightarrow \cdots
\end{gathered}
$$

where $(-)^{\#}$ is the Pontrjagin duality functor from discrete 2-torsion abelian groups to 2-profinite abelian groups.

This may be used to calculate $v_{1}^{-1} \pi_{*} X$ from $K O^{*}\left(\Phi_{1} X ; \hat{\mathbb{Z}}_{2}\right)$ up to extension. To approach $K O^{*}\left(\Phi_{1} X ; \hat{\mathbb{Z}}_{2}\right)$ or $K^{*}\left(\Phi_{1} X ; \hat{\mathbb{Z}}_{2}\right)$, we require:

Definition 9.3 (The $K / 2_{*}$-durable spaces). Following $[8,7.8]$, we say that a space $X$ is $K / 2_{*}$-durable when the $K / 2_{*}$-localization $X \rightarrow X_{K / 2}$ induces an equivalence $\Phi_{1} X \simeq \Phi_{1} X_{K / 2}$ (or equivalently induces an isomorphism $v_{1}^{-1} \pi_{*} X \cong v_{1}^{-1} \pi_{*} X_{K / 2}$ ), and we recall that each connected $H$-space is $K / 2_{*}$-durable. For such $X$, we may apply our key result on $K / 2_{*}$-localizations (Theorem 8.6) to deduce:

Theorem 9.4. If $X$ is a connected $K / 2_{*}$-durable space (e.g. H-space) with a strict isomorphism $\hat{L} M \cong K_{C R}^{*}\left(X ; \hat{\mathbb{Z}}_{2}\right)$ for a strong module $M \in \hat{\mathcal{M}} \Delta$, then there is a (co)fiber sequence of spectra $\Phi_{1} X \rightarrow \mathcal{E} \bar{M} \stackrel{\epsilon}{\rightarrow} \mathcal{E} \bar{\rho} \bar{M}$ such that $\epsilon^{*}: K_{C R}^{*}\left(\mathcal{E} \bar{\rho} \bar{M} ; \hat{\mathbb{Z}}_{2}\right) \rightarrow$ $K_{C R}^{*}\left(\mathcal{E} \bar{M} ; \hat{\mathbb{Z}}_{2}\right)$ is given by $C R^{-1} \theta: C R^{-1} \bar{\rho} \bar{M} \rightarrow C R^{-1} \bar{M}$.

Here, the map $\theta: \bar{\rho} \bar{M} \rightarrow \bar{M}$ is given by

$$
\theta=(\theta, \theta, \theta):\left\{\bar{M}_{C}, \bar{M}_{R}+\bar{M}_{H}, \bar{M}_{R} \cap \bar{M}_{H}\right\} \longrightarrow\left\{\bar{M}_{C}, \bar{M}_{R}, \bar{M}_{H}\right\}
$$

in $\hat{\mathcal{A}} \Delta$. This theorem will be proved below and may be used to calculate $K^{*}\left(\Phi_{1} X\right.$; $\left.\hat{\mathbb{Z}}_{2}\right)$ and $K O^{*}\left(\Phi_{1} X ; \hat{\mathbb{Z}}_{2}\right)$ since it immediately implies:

Theorem 9.5. For $X$ as in Theorem 9.4, there is a $K^{*}\left(-; \hat{\mathbb{Z}}_{2}\right)$ cohomology exact sequence

$$
0 \longrightarrow K^{-2}\left(\Phi_{1} X ; \hat{\mathbb{Z}}_{2}\right) \longrightarrow \bar{M}_{C} \stackrel{\theta}{\longrightarrow} \bar{M}_{C} \longrightarrow K^{-1}\left(\Phi_{1} X ; \hat{\mathbb{Z}}_{2}\right) \longrightarrow 0
$$

and there is a $K O^{*}\left(-; \hat{\mathbb{Z}}_{2}\right)$ cohomology exact sequence

$$
\begin{aligned}
& 0 \longrightarrow K O^{-8}\left(\Phi_{1} X ; \hat{\mathbb{Z}}_{2}\right) \longrightarrow \bar{M}_{C} /\left(\bar{M}_{R}+\bar{M}_{H}\right) \stackrel{\theta}{\longrightarrow} \bar{M}_{C} / \bar{M}_{R} \longrightarrow \\
& K O^{-7}\left(\Phi_{1} X ; \hat{\mathbb{Z}}_{2}\right) \longrightarrow 0 \longrightarrow \bar{M}_{H} /\left(\bar{M}_{R} \cap \bar{M}_{H}\right) \longrightarrow K O^{-6}\left(\Phi_{1} X ; \hat{\mathbb{Z}}_{2}\right) \longrightarrow \\
& \bar{M}_{R} \cap \bar{M}_{H} \stackrel{\theta}{\longrightarrow} \bar{M}_{H} \longrightarrow K O^{-5}\left(\Phi_{1} X ; \hat{\mathbb{Z}}_{2}\right) \longrightarrow 0 \longrightarrow 0 \longrightarrow K O^{-4}\left(\Phi_{1} X ; \hat{\mathbb{Z}}_{2}\right) \longrightarrow \\
& \bar{M}_{C} /\left(\bar{M}_{R} \cap \bar{M}_{H}\right) \stackrel{\theta}{\longrightarrow} \bar{M}_{C} / \bar{M}_{H} \longrightarrow K O^{-3}\left(\Phi_{1} X ; \hat{\mathbb{Z}}_{2}\right) \longrightarrow \\
& \left(\bar{M}_{R}+\bar{M}_{H}\right) /\left(\bar{M}_{R} \cap \bar{M}_{H}\right) \stackrel{\theta}{\longrightarrow} \bar{M}_{R} /\left(\bar{M}_{R} \cap \bar{M}_{H}\right) \longrightarrow \\
& K O^{-2}\left(\Phi_{1} X ; \hat{\mathbb{Z}}_{2}\right) \longrightarrow \bar{M}_{R}+\bar{M}_{H} \stackrel{\theta}{\longrightarrow} \bar{M}_{R} \longrightarrow K O^{-1}\left(\Phi_{1} X ; \hat{\mathbb{Z}}_{2}\right) \longrightarrow 0 \text {. }
\end{aligned}
$$


In these sequences, $\theta$ may be replaced by $\lambda^{2}=-\theta$. Also, for $i, k \in \mathbb{Z}$ with $k$ odd, the Adams operation $\psi^{k}$ in $K^{2 i-1}\left(\Phi_{1} X ; \hat{\mathbb{Z}}_{2}\right), K^{2 i-2}\left(\Phi_{1} X ; \hat{\mathbb{Z}}_{2}\right), K O^{2 i-1}\left(\Phi_{1} X ; \hat{\mathbb{Z}}_{2}\right)$, or $K O^{2 i-2}\left(\Phi_{1} X ; \hat{\mathbb{Z}}_{2}\right)$ agrees with $k^{-i} \psi^{k}$ in the adjacent $\bar{M}$ terms.

Thus, for $X$ as in Theorem 9.4, we may essentially calculate $v_{1}^{-1} \pi_{*} X$ from $\bar{M}$ (up to extension problems) using Theorems 9.2 and 9.5. By $[\mathbf{1 0}, 7.6]$, this approach to $v_{1}^{-1} \pi_{*} X$ may be extended to various other important spaces $X$ using:

Definition 9.6 (The $\widehat{K} \Phi_{1}$-goodness condition). For a space $X$, we let $\Phi_{1}: \widetilde{K}_{C R}^{*}(X$; $\left.\hat{\mathbb{Z}}_{2}\right) \longrightarrow K_{C R}^{*}\left(\Phi_{1} X ; \hat{\mathbb{Z}}_{2}\right)$ denote the $v_{1}$-stabilization homomorphism of $[\mathbf{1 0}, 7.1]$, and we recall that it induces a homomorphism $\Phi_{1}: \hat{Q} K_{\Delta}^{n}\left(X ; \hat{\mathbb{Z}}_{2}\right) / \theta \longrightarrow K_{\Delta}^{n}\left(\Phi_{1} X ; \hat{\mathbb{Z}}_{2}\right)$ in $\hat{\mathcal{A}} \Delta$ for $n=-1,0$ by $[\mathbf{1 0}, 7.4]$, where $\hat{Q} K_{\Delta}^{n}\left(X ; \hat{\mathbb{Z}}_{2}\right) / \theta$ is as in Remark 4.10 and Definition 6.1. Following $[\mathbf{1 0}, 7.5]$, we say that a space $X$ is $\widehat{K} \Phi_{1}$-good when the complex $v_{1}$-stabilization homomorphism $\Phi_{1}: \hat{Q} K^{n}\left(X ; \hat{\mathbb{Z}}_{2}\right) / \theta \rightarrow K^{n}\left(\Phi_{1} X ; \hat{\mathbb{Z}}_{2}\right)$ is an isomorphism for $n=-1,0$. Our next theorem will provide initial examples of $\widehat{K} \Phi_{1^{-}}$ good spaces from which other examples may be built.

Theorem 9.7. If $X$ is a connected $K / 2_{*}$-durable space (e.g. H-space) with a strict isomorphism $\hat{L} M \cong K_{C R}^{*}\left(X ; \hat{\mathbb{Z}}_{2}\right)$ for a strong module $M \in \hat{\mathcal{M}} \Delta$ such that $\theta: \bar{M}_{C} \rightarrow$ $\bar{M}_{C}$ is monic, then $X$ is $\widehat{K} \Phi_{1}$-good with $K^{0}\left(\Phi_{1} X ; \hat{\mathbb{Z}}_{2}\right)=0$, with $K^{-1}\left(\Phi_{1} X ; \hat{\mathbb{Z}}_{2}\right)=$ $\bar{M}_{C} / \theta$, and with $K_{\Delta}^{-1}\left(\Phi_{1} X ; \hat{\mathbb{Z}}_{2}\right) \cong \bar{M} / \theta$.

To prove Theorems 9.4 and 9.7, we first consider the spectrum $\tilde{\mathcal{E}} N$ for a torsionfree exact module $N \in \hat{\mathcal{A}} \Delta$ and note that $\Phi_{1} \Omega^{\infty} \tilde{\mathcal{E}} N \simeq(\tilde{\mathcal{E}} N)_{K / 2} \simeq \mathcal{E} N$.

Lemma 9.8. The space $\Omega^{\infty} \tilde{\mathcal{E}} N$ is $\widehat{K} \Phi_{1}$-good, and the $v_{1}$-stabilization gives a natural isomorphism

$$
\Phi_{1}: \hat{Q} K_{\Delta}^{-1}\left(\Omega^{\infty} \tilde{\mathcal{E}} N ; \hat{\mathbb{Z}}_{2}\right) / \theta \cong K_{\Delta}^{-1}\left(\mathcal{E} N ; \hat{\mathbb{Z}}_{2}\right)
$$

Proof. By [10, 7.1], the homomorphism $\Phi_{1}: K_{\Delta}^{-1}\left(\Omega^{\infty} \tilde{\mathcal{E}} N ; \hat{\mathbb{Z}}_{2}\right) \rightarrow K_{\Delta}^{-1}\left(\mathcal{E} N ; \hat{\mathbb{Z}}_{2}\right)$ is left inverse to the infinite suspension homomorphism, and the lemma now follows by Theorem 6.7 together with Lemma 4.11, and Definition 6.3.

Proof of Theorem 9.4. Applying the functor $\Phi_{1}$ to the fiber sequence of Theorem 8.6, we obtain a (co)fiber sequence of spectra

$$
\Phi_{1} X_{K / 2} \longrightarrow \Phi_{1} \Omega^{\infty} \tilde{\mathcal{E}} \bar{M} \stackrel{\Phi_{1} f}{\longrightarrow} \Phi_{1} \Omega^{\infty} \tilde{\mathcal{E}} \bar{\rho} \bar{M}
$$

for some companion map $f$ of $M$. We then deduce that $\Phi_{1} f$ corresponds to a map $\mathcal{E} \bar{M} \rightarrow \mathcal{E} \bar{\rho} \bar{M}$ having the desired properties by Lemmas 9.8 and 5.4.

Proof of Theorem 9.7. The results on $K^{*}\left(\Phi_{1} X ; \hat{\mathbb{Z}}_{2}\right)$ and $K_{\Delta}^{-1}\left(\Phi_{1} X ; \hat{\mathbb{Z}}_{2}\right)$ follow from Theorem 9.5. Since $K^{*}\left(X ; \hat{\mathbb{Z}}_{2}\right) \cong \hat{\Lambda} M_{C}$ by Lemma 4.6, we obtain isomorphisms $\hat{Q} K^{0}\left(X ; \hat{\mathbb{Z}}_{2}\right) / \theta=0$ and $\hat{Q} K^{-1}\left(X ; \hat{\mathbb{Z}}_{2}\right) / \theta \cong M_{C} / \theta$, and we deduce that $\Phi_{1}: \hat{Q} K^{n}(X ;$ $\left.\hat{\mathbb{Z}}_{2}\right) / \theta \cong K^{n}\left(\Phi_{1} X ; \hat{\mathbb{Z}}_{2}\right)$ for $n=-1,0$ by Lemma 9.8 and naturality. 


\section{Applications to simply-connected compact Lie groups}

We now apply the preceding results to a simply-connected compact Lie group $G$. We first use the representation theory of $G$ to functorially determine the united 2adic $K$-cohomology ring $K_{C R}^{*}\left(G ; \hat{\mathbb{Z}}_{2}\right)=\left\{K^{*}\left(G ; \hat{\mathbb{Z}}_{2}\right), K O^{*}\left(G ; \hat{\mathbb{Z}}_{2}\right)\right\}$ in Theorem 10.3. Then, with slight restrictions on the group, we use the representation theory of $G$ to give expressions for the $K / 2_{*}$-localization $G_{K / 2}$, for the $v_{1}$-stabilization $\Phi_{1} G$, and for the cohomology $K O^{*}\left(\Phi_{1} G ; \hat{\mathbb{Z}}_{2}\right)$, and we also show that $G$ is $\widehat{K} \Phi_{1}$-good. Our results are summarized in Theorem 10.6 and permit calculations of the 2-primary $v_{1}$-periodic homotopy groups $v_{1}^{-1} \pi_{*} G$ using Theorem 9.2, as accomplished very successfully by Davis [14]. In this section, we assume some general familiarity with the representation rings of our Lie groups as described in [12, Sections II.6 and VI.4] and [14, Theorem 2.3].

Definition 10.1 (The representation ring $R_{\Delta} G$ ). For a simply-connected compact Lie group $G$, we let $R G$ be the complex representation ring and let $R_{R} G, R_{H} G \subset$ $R G$ be the real and quaternionic parts of $R G$ with the usual $\lambda$-ring structures on $R G$ and $R_{R} G \oplus R_{H} G$. We also let $t=\psi^{-1}: R G \cong R G, c: R_{R} G \subset R G, r: R G \rightarrow$ $R_{R} G, c^{\prime}: R_{H} G \subset R G$, and $q: R G \rightarrow R_{H} G$ be the usual operations satisfying the $\Delta$ module relations of Definition 4.1. These structures are compatible in the expected ways and combine to give a $\Delta \lambda$-ring $R_{\Delta} G=\left\{R G, R_{R} G, R_{H} G\right\}$ in the sense of $[\mathbf{1 0}$, 6.2]. We let $\tilde{R}_{\Delta} G=\left\{\tilde{R} G, \tilde{R}_{R} G, \tilde{R}_{H} G\right\}$ be the augmentation ideal of $R_{\Delta} G$ given by the kernel $\tilde{R} G$ of the complex augmentation $\operatorname{dim}: R G \rightarrow \mathbb{Z}$, where $\tilde{R}_{R} G=R_{R} G \cap$ $\tilde{R} G$ and $\tilde{R}_{H} G=R_{H} G \cap \tilde{R} G$. We also let $Q R_{\Delta} G=\left\{Q R G, Q R_{R} G, Q R_{H} G\right\}$ be the indecomposables of $R_{\Delta} G$ given by

$$
\begin{gathered}
Q R G=\tilde{R} G /(\tilde{R} G)^{2}, \\
Q R_{R} G=\tilde{R}_{R} G /\left(\left(\tilde{R}_{R} G\right)^{2}+\left(\tilde{R}_{H} G\right)^{2}+r(\tilde{R} G)^{2}\right), \\
Q R_{H} G=\tilde{R}_{H} G /\left(\left(\tilde{R}_{R} G\right)\left(\tilde{R}_{H} G\right)+q(\tilde{R} G)^{2}\right) .
\end{gathered}
$$

It is straightforward to show that $\tilde{R}_{\Delta} G$ and $Q R_{\Delta} G$ inherit $\Delta \lambda$-ring structures (without identities) from $R_{\Delta} G$. Since $Q R_{\Delta} G$ is a $\Delta \lambda$-ring with trivial multiplication, it is equipped with additive operations $t: Q R G \cong Q R G, c: Q R_{R} G \rightarrow Q R G$, $r: Q R G \rightarrow Q R_{R} G, \quad c^{\prime}: Q R_{H} G \rightarrow Q R G, \quad q: Q R G \rightarrow Q R_{H} G, \quad \theta=-\lambda^{2}: Q R G \rightarrow$ $Q R G, \quad \theta=-\lambda^{2}: Q R_{R} G \rightarrow Q R_{R} G, \quad \theta=-\lambda^{2}: Q R_{H} G \rightarrow Q R_{R} G, \quad \psi^{k}: Q R G \rightarrow$ $Q R G, \psi^{k}: Q R_{R} G \rightarrow Q R_{R} G$, and $\psi^{k}: Q R_{H} G \rightarrow Q R_{H} G$ for the odd $k \in \mathbb{Z}$. We now let $\hat{Q} R_{\Delta} G=\left\{\hat{Q} R G, \hat{Q} R_{R} G, \hat{Q} R_{H} G\right\}$ be the 2 -adic completion of $Q R_{\Delta} G$ with the induced additive operations on the components $\hat{Q} R G=\hat{\mathbb{Z}}_{2} \otimes Q R G, \hat{Q} R_{R} G=$ $\hat{\mathbb{Z}}_{2} \otimes Q R_{R} G$, and $\hat{Q} R_{H} G=\hat{\mathbb{Z}}_{2} \otimes Q R_{H} G$.

Lemma 10.2. For a simply-connected compact Lie group $G, \hat{Q} R_{\Delta} G$ is a robust 2-adic Adams $\Delta$-module.

This will be proved below. To determine the cohomology $\operatorname{ring} K_{C R}^{*}\left(G ; \hat{\mathbb{Z}}_{2}\right)=$ $\left\{K^{*}\left(G ; \hat{\mathbb{Z}}_{2}\right), K O^{*}\left(G ; \hat{\mathbb{Z}}_{2}\right)\right\}$ from the representation theory of $G$, we now let $\beta: \hat{Q} R_{\Delta} G$ $\rightarrow \widetilde{K}_{\Delta}^{-1}\left(G ; \hat{\mathbb{Z}}_{2}\right)$ be the 2 -adic Adams $\Delta$-module homomorphism induced by the composition of the canonical homomorphisms $\tilde{R}_{\Delta} G \rightarrow \widetilde{K}_{\Delta}^{0}\left(B G ; \hat{\mathbb{Z}}_{2}\right) \rightarrow \widetilde{K}_{\Delta}^{-1}\left(G ; \hat{\mathbb{Z}}_{2}\right)$. 
Theorem 10.3. For a simply-connected compact Lie group $G$, there is a natural strict isomorphism $\bar{\beta}: \hat{L}\left(\hat{Q} R_{\Delta} G\right) \cong K_{C R}^{*}\left(G ; \hat{\mathbb{Z}}_{2}\right)$.

Proof. This follows by Lemma 10.2 and Theorem 4.9 since $\beta: \hat{Q} R G \rightarrow K^{-1}\left(G ; \hat{\mathbb{Z}}_{2}\right)$ induces an isomorphism $\hat{\Lambda}(\hat{Q} R G) \cong K^{*}\left(G ; \hat{\mathbb{Z}}_{2}\right)$ by $[\mathbf{1 7}]$.

We note that $K_{C R}^{*}\left(G ; \hat{\mathbb{Z}}_{2}\right)$ has a simple system of generators (see Definition 3.3) consisting of the $\beta \tilde{z}_{\gamma} \in K^{-1}\left(G ; \hat{\mathbb{Z}}_{2}\right)$, the $\beta \tilde{x}_{\alpha} \in K O^{-1}\left(G ; \hat{\mathbb{Z}}_{2}\right)$, and the $\beta \tilde{y}_{\beta} \in$ $K O^{-5}\left(G ; \hat{\mathbb{Z}}_{2}\right)$ obtained from the analysis of $\hat{Q} R_{\Delta} G$ below in Remark 10.7 . Thus, by Proposition $3.4, K_{C R}^{*}\left(G ; \hat{\mathbb{Z}}_{2}\right)$ is a free 2-adic $C R$-module on the associated products. However, our description of $K_{C R}^{*}\left(G ; \hat{\mathbb{Z}}_{2}\right)$ as $\hat{L}\left(\hat{Q} R_{\Delta} G\right)$ is more natural and includes the full multiplicative structure. Moreover, it will let us apply our main results to $G$.

Lemma 10.4. For a simply-connected compact Lie group $G$, the 2-adic Adams $\Delta$ module $\hat{Q} R_{\Delta} G$ is regular with $\theta: \hat{Q} R G \rightarrow \hat{Q} R G$ monic.

Proof. This follows by Lemmas 7.9 and 7.10 since $\beta: \hat{Q} R G \rightarrow \widetilde{K}^{-1}\left(G ; \hat{\mathbb{Z}}_{2}\right)$ is monic by Theorem 10.3 .

Thus, $\hat{Q} R_{\Delta} G$ is strong (robust, $\psi^{3}$-splittable, and regular) if and only if it is $\psi^{3}$-splittable, and this is usually the case by:

Lemma 10.5. For a simply-connected compact simple Lie group $G$, the 2-adic Adams $\Delta$-module $\hat{Q} R_{\Delta} G$ is $\psi^{3}$-splittable (and hence strong) if and only if $G$ is not $E_{6}$ or $\operatorname{Spin}(4 k+2)$ with $k$ not a 2-power.

This will be proved below using work of Davis [14]. For a simply-connected compact Lie group $G$, we now let $\hat{Q}_{\Delta}=\left\{\hat{Q}, \hat{Q}_{R}, \hat{Q}_{H}\right\}$ briefly denote the associated stable 2-adic Adams $\Delta$-module $\overline{\hat{Q}_{\Delta} R G}=\left(\hat{Q}_{\Delta} R G\right) / \bar{\phi}$. This agrees with the notation of $[\mathbf{1 0}, 9.2]$ and $[\mathbf{1 4}]$, since our $\hat{Q}_{\Delta}=\left\{\hat{Q}, \hat{Q}_{R}, \hat{Q}_{H}\right\}$ is the 2-adic completion of their $Q_{\Delta}=\left\{Q, Q_{R}, Q_{H}\right\}$. Our main results now give the following omnibus theorem, whose four parts may be expanded in the obvious ways to match the cited theorems.

Theorem 10.6. Let $G$ be a simply-connected compact Lie group such that the 2adic Adams $\Delta$-module $\hat{Q}_{\Delta} R G$ is $\psi^{3}$-splittable (see Lemma 10.5), and let $\hat{Q}_{\Delta}=$ $\left\{\hat{Q}, \hat{Q}_{R}, \hat{Q}_{H}\right\}$ be the associated stable 2-adic Adams $\Delta$-module. Then:

(i) the $K / 2_{*}$-localization $G_{K / 2}$ is the homotopy fiber of a map $\Omega^{\infty} \tilde{\mathcal{E}} \hat{Q}_{\Delta} \rightarrow$ $\Omega^{\infty} \tilde{\mathcal{E}} \bar{\rho} \hat{Q}_{\Delta}$ with low dimensional modifications as in Theorem 8.6;

(ii) the 2-adic $v_{1}$-stabilization $\Phi_{1} G$ is the homotopy fiber of a map of spectra $\mathcal{E} \hat{Q}_{\Delta} \rightarrow \mathcal{E} \bar{\rho} \hat{Q}_{\Delta}$ as in Theorem 9.4;

(iii) there is an exact sequence

$$
0 \longrightarrow K O^{-8}\left(\Phi_{1} G ; \hat{\mathbb{Z}}_{2}\right) \longrightarrow \hat{Q} /\left(\hat{Q}_{R}+\hat{Q}_{H}\right) \stackrel{\theta}{\longrightarrow} \hat{Q} / \hat{Q}_{R} \longrightarrow \cdots
$$

continuing as in Theorem 9.5;

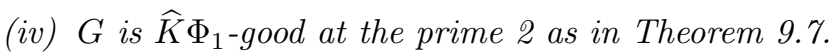


The exact sequence in (iii) permits calculations of the 2-primary $v_{1}$-periodic homotopy groups $v_{1}^{-1} \pi_{*} G$ using Theorem 9.2 as accomplished by Davis [14]. This exact sequence was previously obtained in [10, Theorem 9.3] using indirect algebraic methods under the hard-to-verify condition that $G$ was $\widehat{K} \Phi_{1}$-good. It is now obtained using the $K O^{*}\left(-; \hat{\mathbb{Z}}_{2}\right)$ cohomology exact sequence of the (co)fiber sequence in (ii) under an accessible algebraic condition that implies the $\widehat{K} \Phi_{1}$-goodness of $G$ by (iv).

We devote the rest of the section to proving Lemmas 10.2 and 10.5 using:

Remark 10.7 (Generators for representation rings). For a simply-connected compact Lie group $G$, standard results summarized in [14, Theorem 2.3] show that $R G$ is a finitely generated polynomial ring $\mathbb{Z}\left[z_{\gamma}, z_{\gamma}^{*}, x_{\alpha}, y_{\beta}\right]_{\gamma, \alpha, \beta}$ on certain basic complex representations $z_{\gamma}$ together with their conjugates $z_{\gamma}^{*}=t z_{\gamma}$, certain basic real representations $x_{\alpha}$, and certain basic quaternionic representations $y_{\beta}$. Moreover, in terms of these generators, the $\mathbb{Z} / 2$-graded ring $\left\{R_{R} G, R_{H} G\right\}$ is characterized by the fact that its quotient $\left\{R_{R} G / r R G, R_{H} G / q R G\right\}$ is a $\mathbb{Z} / 2$-graded polynomial algebra $\mathbb{Z} / 2\left[x_{\alpha}, \bar{\phi} z_{\gamma}, y_{\beta}\right]_{\alpha, \gamma, \beta}$ on the real generators $x_{\alpha}$ and $\bar{\phi} z_{\gamma}$ (with $c \bar{\phi} z_{\gamma}=z_{\gamma}^{*} z_{\gamma}$ ) and the quaternionic generators $y_{\beta}$. Consequently, the indecomposables $Q R_{\Delta} G=$ $\left\{Q R G, Q R_{R} G, Q R_{H} G\right\}$ may be expressed as

$$
\begin{aligned}
Q R G & =\mathbb{Z}\left\{\tilde{z}_{\gamma}, \tilde{z}_{\gamma}^{*}, c \tilde{x}_{\alpha}, c^{\prime} \tilde{y}_{\beta}\right\}_{\gamma, \alpha, \beta}, \\
Q R_{R} G & =\mathbb{Z}\left\{r \tilde{z}_{\gamma}, \tilde{x}_{\alpha}, r c^{\prime} \tilde{y}_{\beta}\right\}_{\gamma, \alpha, \beta} \oplus \mathbb{Z} / 2\left\{\bar{\phi} \tilde{z}_{\gamma}\right\}_{\gamma}, \\
Q R_{H} G & =\mathbb{Z}\left\{q \tilde{z}_{\gamma}, q c \tilde{x}_{\alpha}, \tilde{y}_{\beta}\right\}_{\gamma, \alpha, \beta}
\end{aligned}
$$

where $\tilde{w}$ denotes $w-\operatorname{dim} w$ for $w \in R G$. Thus, the 2-adic indecomposables $\hat{Q} R_{\Delta} G$ $=\left\{\hat{Q} R G, \hat{Q} R_{R} G, \hat{Q} R_{H} G\right\}$ may be expressed similarly using $\hat{\mathbb{Z}}_{2}$ in place of $\mathbb{Z}$, and the stable 2-adic indecomposables $\hat{Q}_{\Delta}=\left\{\hat{Q}, \hat{Q}_{R}, \hat{Q}_{H}\right\}$ may be expressed as

$$
\begin{aligned}
\hat{Q} & =\hat{\mathbb{Z}}_{2}\left\{\tilde{z}_{\gamma}, \tilde{z}_{\gamma}^{*}, c \tilde{x}_{\alpha}, c^{\prime} \tilde{y}_{\beta}\right\}_{\gamma, \alpha, \beta}, \\
\hat{Q}_{R} & =\hat{\mathbb{Z}}_{2}\left\{r \tilde{z}_{\gamma}, \tilde{x}_{\alpha}, r c^{\prime} \tilde{y}_{\beta}\right\}_{\gamma, \alpha, \beta}, \\
\hat{Q}_{H} & =\hat{\mathbb{Z}}_{2}\left\{q \tilde{z}_{\gamma}, q c \tilde{x}_{\alpha}, \tilde{y}_{\beta}\right\}_{\gamma, \alpha, \beta} .
\end{aligned}
$$

Proof of Lemma 10.2. Since $Q R_{\Delta} G$ is a $\Delta \lambda$-ring with trivial multiplication, it is straightforward to check all of the required relations for operations (see Definitions 4.3 and 6.1). In particular, we deduce $\theta \theta r=\theta r \theta$ from the relations $\lambda^{4} r=$ $r \lambda^{4}+\bar{\phi} \lambda^{2}, \lambda^{4}=-\lambda^{2} \lambda^{2}, \bar{\phi}=\lambda^{2} r-r \lambda^{2}, 2 \bar{\phi}=0$, and $\theta=-\lambda^{2}$, which hold generally in $\Delta \lambda$-rings with trivial multiplication $[\mathbf{1 0}, 6.2]$. We next observe that $\hat{Q} R G$, $\hat{Q} R_{R} G$, and $\hat{Q} R_{H} G$ are stable 2-adic Adams modules by [6, 6.2], since $Q R G$ and $Q R_{R} G \oplus Q R_{H} G$ are $\gamma$-nilpotent and finitely generated abelian (because they have trivial multiplications and have finite generating sets of elements $\tilde{w}$ for representations $w$ ). Thus, $\hat{Q} R_{\Delta} G$ is a 2 -adic Adams $\Delta$-module, and it must be robust by the analysis of Remark 10.7.

To check the $\psi^{3}$-splittability of $\hat{Q} R_{\Delta} G$, we let $h G=\operatorname{ker}(1-t) / \operatorname{im}(1+t)$ be the augmented algebra over $\mathbb{Z} / 2$ obtained from $R G$ using the involution $t=\psi^{-1}: R G \cong$ 
$R G$. This is a polynomial algebra $h G \cong \mathbb{Z} / 2\left[c \tilde{x}_{\alpha}, \tilde{z}_{\gamma}^{*} \tilde{z}_{\gamma}, c^{\prime} \tilde{y}_{\beta}\right]_{\alpha, \gamma, \beta}$ which is $\mathbb{Z} / 2$-graded, since there is an isomorphism

$$
c+c^{\prime}: R_{R} G / r R G \oplus R_{H} G / q R G \cong h G,
$$

and we let $Q_{R} h G \cong \mathbb{Z} / 2\left\{c \tilde{x}_{\alpha}, \tilde{z}_{\gamma}^{*} \tilde{z}_{\gamma}\right\}_{\alpha, \gamma}$ denote the real (degree 0) indecomposables. We define a homomorphism $s: Q R G \rightarrow Q_{R} h G$ by $s[u]=\left[u^{*} u\right]$ for $u \in \tilde{R} G$ and note that $s Q R G=\mathbb{Z} / 2\left\{\tilde{z}_{\gamma}^{*} \tilde{z}_{\gamma}\right\}_{\gamma}$. We view $s$ as a homomorphism of $\psi^{3}$-modules (abelian groups with endomorphisms $\left.\psi^{3}\right)$ as in [14, 2.4].

Lemma 10.8. For a simply-connected compact Lie group $G, \hat{Q} R_{\Delta} G$ is $\psi^{3}$-splittable if and only if the $\psi^{3}$-submodule $s Q R G \subset Q_{R} h G$ is a direct summand.

Proof. By Definition 7.2 and the proof of Lemma 7.3, $\hat{Q} R_{\Delta} G$ is $\psi^{3}$-splittable if and only if the $\psi^{3}$-submodule $\bar{\phi} \hat{Q} R G \subset \hat{Q} R_{R} G / r \hat{Q} R G$ (or equivalently $\bar{\phi} Q R G \subset$ $\left.Q R_{R} G / r Q R G\right)$ is a direct summand. The lemma now follows since $\bar{\phi} Q R G$ corresponds to $s Q R G$ under the isomorphism $c: Q R_{R} G / r Q R G \cong Q_{R} h G$.

Proof of Lemma 10.5. By Lemma 10.8 and Davis [14, Theorem 1.3], the following conditions are successively equivalent: $\hat{Q} R_{\Delta} G$ is $\psi^{3}$-splittable; the $\psi^{3}$-submodule $s Q R G \subset Q_{R} h G$ is a direct summand; $G$ satisfies the Technical Condition of [14, Definition 2.4]; $G$ is not $E_{6}$ or $\operatorname{Spin}(4 k+2)$ with $k$ not a 2-power.

\section{Proofs of basic lemmas for $\hat{L}$}

We shall prove Lemmas 4.5, 4.6, and 4.11 showing the basic properties of the functor $\hat{L}: \theta \Delta \hat{\mathcal{M}}$ od $\rightarrow \phi \mathcal{C} \mathcal{R} \hat{\mathcal{A}} l g$, where $\theta \Delta \hat{\mathcal{M}}$ od is the category of 2-adic $\theta \Delta$-modules and $\phi \mathcal{C R} \hat{\mathcal{A}} l g$ is that of special 2-adic $\phi C R$-algebras (see Definitions 4.3 and 3.2). We first introduce an intermediate category of modules.

Definition 11.1 (The 2-adic $\eta \Delta$-modules). By a 2-adic $\eta \Delta$-module $N=\left\{N_{C}, N_{R}\right.$, $\left.N_{H}, N_{S}\right\}$, we mean a 2-adic $\Delta$-module $\left\{N_{C}, N_{R}, N_{H}\right\}$, with operations $t, c, r, c^{\prime}$, and $q$ as in Definition 4.1, together with a 2-profinite abelian group $N_{S}$ and continuous additive operations $\bar{\phi}: N_{C} \rightarrow N_{R}, \eta: N_{R} \rightarrow N_{S},()^{[2]}: N_{R} \rightarrow N_{S}$, and () $)^{[2]}: N_{H} \rightarrow$ $N_{S}$ satisfying the following relations for elements $z \in N_{C}, x \in N_{R}$, and $y \in N_{H}$ :

$$
\begin{aligned}
& \bar{\phi} c x=0, \quad \bar{\phi} c^{\prime} y=0, \quad \bar{\phi} t z=\bar{\phi} z, \quad 2 \bar{\phi} z=0, \quad c \bar{\phi} z=0, \\
& (\bar{\phi} z)^{[2]}=0, \quad 2 \eta x=0, \quad \eta r z=0, \quad(q z)^{[2]}=(r z)^{[2]}=\eta \bar{\phi} z .
\end{aligned}
$$

We let $\eta \Delta \hat{\mathcal{M}}$ od denote the category of 2 -adic $\eta \Delta$-modules.

Remark 11.2 (A functorial interpretation of admissible maps). Let $J: \theta \Delta \hat{\mathcal{M}}$ od $\rightarrow$ $\eta \Delta \hat{\mathcal{M}}$ od be the functor carrying a 2 -adic $\theta \Delta$-module $M$ to the 2 -adic $\eta \Delta$-module $J M=\left\{M_{C}, M_{R}, M_{H}, M_{R} / r M_{C}\right\}$ having the original operations $t, c, r, c^{\prime}, q$, and $\bar{\phi}$ together with operations $\eta: M_{R} \rightarrow M_{R} / r M_{C}, \quad()^{[2]}: M_{R} \rightarrow M_{R} / r M_{C}$, and ()$^{[2]}: M_{H} \rightarrow M_{R} / r M_{C}$ given by $\eta x=[x], x^{[2]}=[\theta x]$, and $y^{[2]}=[\theta y]$ for $x \in M_{R}$ and $y \in M_{H}$. Let $I: \phi \mathcal{C R} \hat{\mathcal{A}} l g \rightarrow \eta \Delta \hat{\mathcal{M}}$ od be the functor carrying a special 2-adic $\phi C R$-algebra $A$ to the 2-adic $\eta \Delta$-module $I A=\left\{\tilde{A}_{C}^{-1}, \tilde{A}_{R}^{-1}, \tilde{A}_{R}^{-5}, \tilde{A}_{R}^{-2}\right\}$ having the 
operations $t, c, r, c^{\prime}$, and $q$ of $\Delta^{-1} \tilde{A}$ (see Definition 4.1) together with operations $\bar{\phi}: \tilde{A}_{C}^{-1} \rightarrow \tilde{A}_{R}^{-1}, \quad \eta: \tilde{A}_{R}^{-1} \rightarrow \tilde{A}_{R}^{-2}, \quad()^{[2]}: \tilde{A}_{R}^{-1} \rightarrow \tilde{A}_{R}^{-2}$, and ()$^{[2]}: \tilde{A}_{R}^{-5} \rightarrow \tilde{A}_{R}^{-2}$ given by $\bar{\phi} z=\eta \phi z, \eta x=\eta x, x^{[2]}=x^{2}$, and $y^{[2]}=B_{R}^{-1} y^{2}$ for $z \in A_{C}^{-1}, x \in A_{R}^{-1}$, and $y \in A_{R}^{-5}$. We now easily see:

Lemma 11.3. For $M \in \theta \Delta \hat{\mathcal{M}}$ od and $A \in \phi C R \hat{\mathcal{A}} l g$, an admissible map $f: M \rightarrow A$ is equivalent to a map $f: J M \rightarrow I A$ in $\eta \Delta \hat{\mathcal{M}}$ od.

To construct the functor $\hat{L}$, we need:

Lemma 11.4. The functor $I: \phi \mathcal{C R} \hat{\mathcal{A}} l g \rightarrow \eta \Delta \hat{\mathcal{M}}$ od has a left adjoint $\hat{V}: \eta \Delta \hat{\mathcal{M}}$ od $\rightarrow \phi \mathcal{C} \mathcal{R} \hat{\mathcal{A}} l g$.

Proof. This follows by the Special Adjoint Functor Theorem (see [19]) since $I$ preserves small limits and since $\phi \mathcal{C} \mathcal{R} \hat{\mathcal{A}} l g$ has a small cogenerating set by Lemma 11.5 below.

A special 2-adic $\phi C R$-algebra $A$ will be called finite when the groups $\tilde{A}_{C}^{m}$ and $\tilde{A}_{R}^{m}$ are finite for all $m$.

Lemma 11.5. Each special 2-adic $\phi C R$-algebra $A$ is the inverse limit of its finite quotients in $\phi \mathcal{C} \mathcal{R} \hat{\mathcal{A}} l g$.

Proof. This is similar to the corresponding result for topological rings in $[\mathbf{2 2}, 5.1 .2]$. For a 2-adic $C R$-submodule $G \subset \tilde{A}$ with $\tilde{A} / G$ finite, we must obtain a special 2-adic $\phi C R$-ideal $H$ of $A$ with $H \subset G$ and $\tilde{A} / H$ finite. We first obtain an ideal $M$ of $A_{R}$ (closed under $B_{R}, B_{R}^{-1}, \eta$, and $\xi$ ) with $M \subset G_{R}$ and $\tilde{A}_{R} / M$ finite as in [22]. We next obtain an ideal $N$ of $A_{C}$ (closed under $B, B^{-1}$, and $t$ ) with $N \subset G_{C} \cap r^{-1} M \cap$ $\phi^{-1} M^{0}$ and $\tilde{A}_{C} / N$ finite as in [22]. The desired ideal $H$ is now given by $H_{C}=N$ and $H_{R}=M \cap c^{-1} N$.

Proof of Lemma 4.5. Using Lemmas 11.3 and 11.4, we obtain the desired universal algebra $\hat{L} M$ from the functor $\hat{L}=\hat{V} J: \theta \Delta \hat{\mathcal{M}}$ od $\rightarrow \phi \mathcal{C} \mathcal{R} \hat{\mathcal{M}}$ od.

A 2-adic $\eta \Delta$-module $N$ is called sharp when $\eta: N_{R} / r N_{C} \rightarrow N_{S}$ is an isomorphism, and we may now derive the properties of $\hat{L}$ from the corresponding properties of $\hat{V}$ on such sharp modules.

Lemma 11.6. For a sharp 2-adic $\eta \Delta$-module $N$, the canonical map $\hat{\Lambda} N_{C} \rightarrow$ $(\hat{V} N)_{C}$ is an algebra isomorphism.

Proof. Let $W: \phi \mathcal{C} \mathcal{R} \hat{\mathcal{A}} l g \rightarrow \mathcal{C} \hat{\mathcal{A}} l g$ be the forgetful functor carrying each $A \in \phi \mathcal{C} \mathcal{R} \hat{\mathcal{A}} l g$ to its complex part $A_{C} \in \mathcal{C} \hat{\mathcal{A}} l g$ where $\mathcal{C} \hat{\mathcal{A}} l g$ is the category of special 2-adic $C$ algebras, which are defined similarly to special 2-adic $\phi C R$-algebras (see Definition 3.2) but using only complex terms and their operations. The functor $W$ has a right adjoint $H: \mathcal{C} \hat{\mathcal{A}} l g \rightarrow \phi \mathcal{C} \mathcal{R} \hat{\mathcal{A}} l g$ where $(H X)_{C}=X$ and $(H X)_{R}=\{x \in X \mid t x=$ $x\}$ with $c=1, \quad r=1+t, \eta=0, \phi z=z^{*} z$ for $z \in X^{0}$, and $\phi w=B^{-1} w^{*} w$ for $w \in X^{-1}$. For each $N \in \eta \Delta \hat{\mathcal{M}}$ od and each $X \in \mathcal{C} \hat{\mathcal{A}} l g$, a map $N \rightarrow I H X$ in $\eta \Delta \hat{\mathcal{M}}$ od corresponds to a map $N_{C} \rightarrow \tilde{X}^{-1}$ respecting $t$, which in turn corresponds to a map $\hat{\Lambda} N_{C} \rightarrow X$ in $\mathcal{C} \hat{\mathcal{A}} l g$. Hence, since $W \hat{V}$ is left adjoint to $I H$, the canonical map $\hat{\Lambda} N_{C} \rightarrow W \hat{V} N$ is an isomorphism. 
Proof of Lemma 4.6. For a 2-adic $\theta \Delta$-module $M$, the canonical map $\hat{\Lambda} M_{C} \rightarrow$ $(\hat{L} M)_{C}$ is an isomorphism by Lemma 11.6 and by the above proof of Lemma 4.5.

Let $\hat{Q}: \phi \mathcal{C R} \hat{\mathcal{A}} l g \rightarrow \phi \mathcal{C} \mathcal{R} \hat{\mathcal{M}}$ od be the functor carrying each $A \in \phi \mathcal{C} \mathcal{R} \hat{\mathcal{A}} l g$ to its indecomposables $\hat{Q} A \in \phi \mathcal{C} \mathcal{R} \hat{\mathcal{M}}$ od where $\phi \mathcal{C R} \hat{\mathcal{M}}$ od is the category of special 2-adic $\phi C R$-modules, which may be defined as the augmentation ideals of the special 2-adic $\phi C R$-algebras having trivial multiplication.

Lemma 11.7. For a sharp 2-adic $\eta \Delta$-module $N$, the canonical map $\left\{N_{C}, N_{R}, N_{H}\right\}$ $\rightarrow \Delta^{-1} \hat{Q} \hat{V} N$ is an isomorphism.

Proof. The functor $\hat{Q}$ has a right adjoint $E: \phi \mathcal{C R} \hat{\mathcal{M}}$ od $\rightarrow \phi \mathcal{C R} \hat{\mathcal{A}} l g$ where $E X=\underline{e} \oplus$ $X$. Since $\hat{Q} \hat{V}: \eta \Delta \hat{\mathcal{M}}$ od $\rightarrow \phi \mathcal{C} \mathcal{R} \hat{\mathcal{M}}$ od is left adjoint to $I E$, a detailed analysis shows that $\hat{Q} \hat{V} N$ is a special 2-adic $\phi C R$-module with $(\hat{Q} \hat{V} N)_{C}^{-1}=N_{C},(\hat{Q} \hat{V} N)_{R}^{-1}=N_{R}$, and $(\hat{Q} \hat{V} N)_{R}^{-5}=N_{H}$.

Proof of Lemma 4.11. For a 2-adic $\theta \Delta$-module $M$, the canonical map $M \rightarrow$ $\Delta^{-1} \hat{Q} \hat{L} M$ is an isomorphism by Lemma 11.7 and the above proof of Lemma 4.5.

\section{Proof of the Bott exactness lemma for $\hat{L}$}

We must now prove Lemma 4.8 showing the Bott exactness of $\hat{L} M$ for a robust 2 -adic $\theta \Delta$-module $M$. This lemma will follow easily from the corresponding result for $\eta \Delta$-modules (Lemma 12.1), whose proof will extend through most of this section. We say that a 2 -adic $\eta \Delta$-module $N$ is profinitely sharp when it is the inverse limit of an inverse system of finite sharp 2-adic $\eta \Delta$-modules. This obviously implies that $N$ is sharp. We call $N$ robust when:

(i) $N$ is profinitely sharp;

(ii) the 2-adic $\Delta$-module $\left\{N_{C}, N_{R} / \bar{\phi} N_{C}, N_{H}\right\}$ is torsion-free and exact;

(iii) $\operatorname{ker} \bar{\phi}=c N_{R}+c^{\prime} N_{H}+2 N_{C}$.

Lemma 12.1. If $N$ is a robust 2-adic $\eta \Delta$-module, then the special 2-adic $\phi C R$ algebra $\hat{V} N$ is Bott exact; in fact, $\hat{V} N$ is the inverse limit of an inverse system of finitely generated free 2-adic CR-modules.

This will be proved at the end of the section.

Proof of Lemma 4.8. For a robust 2-adic $\theta \Delta$-module $M$, the 2-adic $\eta \Delta$-module $J M$ is also robust, and hence $\hat{L} M$ has the required properties by Lemma 12.1 and the proof of Lemma 4.5 in Section 11.

Before proving Lemma 12.1, we must analyze the robust 2 -adic $\eta \Delta$-modules, and we start with:

Definition 12.2 (The complex 2-adic $\eta \Delta$-modules). The functor $(-)_{C}: \eta \Delta \hat{\mathcal{M}}$ od $\rightarrow$ $\hat{\mathcal{A}} b$ from the 2-adic $\eta \Delta$-modules to the 2-profinite abelian groups has a left adjoint $C: \hat{\mathcal{A}} b \rightarrow \eta \Delta \hat{\mathcal{M}}$ od with $C(G)_{C}=G \oplus G=G \oplus t G, C(G)_{R}=G \oplus G / 2=r G \oplus \bar{\phi} G$, $C(G)_{H}=G=q G$, and $C(G)_{S}=G / 2=(\bar{\phi} G)^{[2]}$ for $G \in \hat{\mathcal{A}} b$. A 2-adic $\eta \Delta$-module 
will be called complex when it is isomorphic to $C(G)$ for some $G$. If $G$ is torsionfree, then $C(G)$ is obviously robust. For an arbitrary $N \in \eta \Delta \hat{\mathcal{M}}$ od and $G \in \hat{\mathcal{A}} b$, we may describe the possible maps $N \rightarrow C(G)$ as follows. Let $f: N_{C} \rightarrow G$ and $g: N_{S} \rightarrow G / 2$ be maps such that the diagram

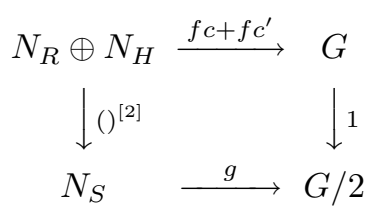

commutes. Then there is a map $F(f, g): N \rightarrow C(G)$ with components $(f, f t): N_{C} \rightarrow$ $G \oplus G, \quad(f c, g \eta): N_{R} \rightarrow G \oplus G / 2, \quad f c^{\prime}: N_{H} \rightarrow G$, and $g: N_{S} \rightarrow G / 2$. Moreover, each map $N \rightarrow C(G)$ is of the above form for some $f$ and $g$. When $N$ is robust, the compatibility condition on $f: N_{C} \rightarrow G$ and $g: N_{S} \rightarrow G / 2$ may be expressed by the commutativity of the diagram

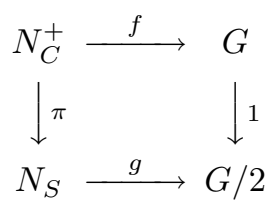

where $N_{C}^{+}=\left\{z \in N_{C} \mid t z=z\right\}$ and $\pi$ is the composition of $\left(c, c^{\prime}\right): N_{R} / \bar{\phi} N_{C} \coprod_{N_{C}} N_{H}$ $\cong N_{C}^{+}$and ()$^{[2]}: N_{R} / \bar{\phi} N_{C} \coprod_{N_{C}} N_{H} \rightarrow N_{S}$. Letting $N_{C}^{-}=\left\{z \in N_{C} \mid t z=-z\right\}$, we now have:

Lemma 12.3. If $\tilde{N} \subset N$ is an inclusion of robust 2-adic $\eta \Delta$-modules such that $N_{C} / \tilde{N}_{C}$ is torsion-free and $\tilde{N}_{C}^{-}=N_{C}^{-}$, then each map $\tilde{N} \rightarrow C(G)$ for $G \in \hat{\mathcal{A}} b$ may be extended to a map $N \rightarrow C(G)$ of 2-adic $\eta \Delta$-modules.

Proof. For a given map $F(\tilde{\tilde{N}}, \tilde{g}): \tilde{N} \rightarrow C(G)$, we first extend $\tilde{g}: \tilde{N}_{S} \rightarrow G / 2$ to a map $g: N_{S} \rightarrow G / 2$. Since $\tilde{N}_{C} / \tilde{N}_{C}^{+} \cong \tilde{N}_{C}^{-}, N_{C} / N_{C}^{+} \cong N_{C}^{-}$, and $\tilde{N}_{C}^{-}=N_{C}^{-}$, we see that $N_{C}$ is the pushout of the inclusions $N_{C}^{+} \leftarrow \tilde{N}_{C}^{+} \rightarrow \tilde{N}_{C}$. Thus, the maps $g \pi: N_{C}^{+} \rightarrow$ $G / 2$ and $[\tilde{f}]: \tilde{N}_{C} \rightarrow G / 2$ induce a map $f^{\prime}: N_{C} \rightarrow G / 2$, and we obtain a commutative diagram

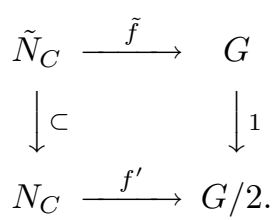

Since $N_{C} / \tilde{N}_{C}$ is projective in $\hat{\mathcal{A}} b$, we may now choose a lifting $f: N_{C} \rightarrow G$ in the diagram, and this gives the desired extension $F(f, g): N_{C} \rightarrow C(G)$ of $F(\tilde{f}, \tilde{g})$.

Lemma 12.4. For a robust 2-adic $\eta \Delta$-module $N$, there exists a decomposition $N \cong$ $C(G) \oplus P$ where $G$ is torsion-free and $P$ is robust with $t=1$ on $P_{C}$.

Proof. By the factorization of positively torsion-free groups in Definition 5.3, there exists a decomposition $N_{C} \cong(G \oplus t G) \oplus H$ with $t=1$ on $H$, and we let $i: C(G) \rightarrow$ 
$N$ be the induced map. Then $i$ is monic since $i:\{G \oplus t G, G, G\} \rightarrow\left\{N_{C}, N_{R} / \bar{\phi} N_{C}\right.$, $\left.N_{H}\right\}$ is monic by [10, Lemma 4.8], and since $i: G / 2 \rightarrow \bar{\phi} N_{C}$ and $\eta: \bar{\phi} N_{C} \rightarrow N_{S}$ are monic by the proof of Lemma 7.3. Thus, $i: C(G) \rightarrow N$ has a left inverse by Lemma 12.3, and the result follows.

Definition 12.5 (The $t$-trivial 2 -adic $\eta \Delta$-modules). A 2 -adic $\eta \Delta$-module $N$ will be called $t$-trivial when $t=1$ on $N_{C}$. When $N$ is $t$-trivial and robust, it must have $\bar{\phi}=0: N_{C} \rightarrow N_{R}$ since $N_{C}=c N_{R}+c^{\prime} N_{H}$ by the exactness of $\left\{N_{C}, N_{R} / \bar{\phi} N_{C}, N_{H}\right\}$. Moreover, it must also have $\left(r N_{C}\right)^{[2]}=0, \quad\left(q N_{C}\right)^{[2]}=0$, and $c+c^{\prime}: N_{R} / r N_{C} \oplus$ $N_{H} / q N_{C} \cong N_{C} / 2$ by [10, Lemma 4.7]. Hence, the operations ()$^{[2]}: N_{R} \rightarrow N_{S}$ and ()$^{[2]}: N_{H} \rightarrow N_{S}$ induce operations $\bar{\theta}: N_{R} / r N_{C} \rightarrow N_{R} / r N_{C}$ and $\bar{\theta}: N_{H} / q N_{C} \rightarrow$ $N_{R} / r N_{C}$, where the $\bar{\theta}$-module $N_{R} / r N_{C}$ is profinite since $N$ is profinitely sharp. In this way, a $t$-trivial robust 2 -adic $\eta \Delta$-module $N$ corresponds to a torsion-free group $G \in \hat{\mathcal{A}} b$ together with a decomposition $(G / 2)_{R} \oplus(G / 2)_{H}=G / 2$ equipped with operations $\bar{\theta}:(G / 2)_{R} \rightarrow(G / 2)_{R}$ and $\bar{\theta}:(G / 2)_{H} \rightarrow(G / 2)_{R}$ such that the $\bar{\theta}$ module $(G / 2)_{R}$ is profinite. We say that a 2-adic $\eta \Delta$-module $N$ is of finite type when $N_{C}, N_{R}, N_{H}$, and $N_{S}$ are finitely generated over $\hat{\mathbb{Z}}_{2}$, and we now easily deduce:

Lemma 12.6. A t-trivial robust 2-adic $\eta \Delta$-module may be expressed as the inverse limit of an inverse system of t-trivial robust quotient modules of finite type.

A similar result obviously holds for the robust 2-adic $\eta \Delta$-modules $C(G)$ with $G$ torsion-free, and the following lemma will now let us restrict our study of $\hat{V}$ to the robust modules of finite type.

Lemma 12.7. If a 2-adic $\eta \Delta$-module $N$ is the inverse limit of an inverse system $\left\{N_{\alpha}\right\}_{\alpha}$ of quotient modules, then $\hat{V} N \cong \lim _{\alpha} \hat{V} N_{\alpha}$.

Proof. For a finite special 2-adic $\phi C R$-algebra $F$, there is a canonical isomorphism $\operatorname{Hom}\left(\lim _{\alpha} \hat{V} N_{\alpha}, F\right) \cong \operatorname{Hom}(\hat{V} N, F)$. Hence the map $\hat{V} N \rightarrow \lim _{\alpha} \hat{V} N_{\alpha}$ is an isomorphism by Lemma 11.5 .

Proof of Lemma 12.1. It now suffices to show that $\hat{V} N$ is a free 2-adic $C R$ module when $N=C(G) \oplus P$ for a finitely generated free $\hat{\mathbb{Z}}_{2}$-module $G$ and a $t$ trivial robust 2-adic $\eta \Delta$-module $P$ of finite type. By Definition 7.1, we may choose finite ordered sets of elements $\left\{z_{k}\right\}_{k}$ in $G,\left\{x_{i}\right\}_{i}$ in $P_{R}$, and $\left\{y_{j}\right\}_{j}$ in $P_{H}$ such that $G$ is a free $\hat{\mathbb{Z}}_{2}$-module on $\left\{z_{k}\right\}_{k}$ and $\left\{P_{C}, P_{R}, P_{H}\right\}$ is a free 2-adic $\Delta$-module on $\left\{x_{i}\right\}_{i}$ and $\left\{y_{j}\right\}_{j}$. Since $P_{S}$ is a free $\mathbb{Z} / 2$-module on the generators $\left\{\eta x_{i}\right\}_{i}$, there are expressions $x_{i}^{[2]}=r_{i}$ and $y_{j}^{[2]}=s_{j}$ for each $i$ and $j$ where the $r_{i}$ and $s_{j}$ are $\mathbb{Z} / 2$-linear combinations of these generators. We may now obtain $\hat{V} N$ as the free augmented 2adic $C R$-algebra on the generators $x_{i} \in(\widehat{\hat{V} N})_{R}^{-1}, y_{j} \in(\widetilde{\hat{V} N})_{R}^{-5}, z_{k} \in(\widehat{\hat{V} N})_{C}^{-1}$, and $\phi z_{k} \in(\widehat{\hat{V} N})_{R}^{0}$ subject to the relations $x_{i}^{2}=r_{i}, y_{j}^{2}=B_{R} s_{j}, z_{k}^{2}=0, z_{k}^{*} z_{k}=B c \phi z_{k}$, and $\left(\phi z_{k}\right)^{2}=0$ for each $i, j$, and $k$. It follows by a straightforward analysis that $\hat{V} N$ is a free 2-adic $C R$-module on the associated products (see Definition 3.3) of $\left\{x_{i}\right\}_{i},\left\{y_{j}\right\}_{j}$, and $\left\{z_{k}\right\}_{k}$. 


\section{Proofs for regular modules}

We first show that our strict nonlinearity condition (see Definition 7.7) for 2-adic Adams modules agrees with that of $[7,2.4]$, and we then prove Lemmas 7.9 and 7.10 for regular modules. For a 2 -adic Adams module $A$, we let $T A \subset A$ be given by the pullback square

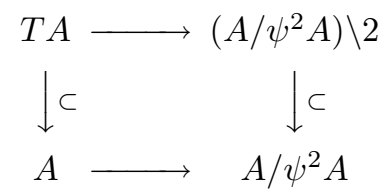

where $\left(A / \psi^{2} A\right) \backslash 2$ is the kernel of $2: A / \psi^{2} A \rightarrow A / \psi^{2} A$. Since the square is also a pushout, $A$ is quasilinear if and only if $T A=A$. Now let $T^{\infty} A$ be the intersection of the submodules $T^{i} A \subset A$ for $i>0$.

Lemma 13.1. $T^{\infty} A$ is the largest quasilinear submodule of $A$, and hence $A_{q l}=$ $T^{\infty} A$.

Proof. Using the inverse limit of the pullback squares for $T^{i} A$ with $i>0$, we find that $T^{\infty} A$ contains each quasilinear submodule of $A$ and that $T\left(T^{\infty} A\right)=T^{\infty} A$.

Remark 13.2 (Strict nonlinearity conditions). Our definition of strict nonlinearity in Section 7 is equivalent to our earlier definition in $[\mathbf{7}, 2.3$ and 2.4]. In fact, for a 2-adic Adams module $A$, the largest quasilinear submodule $A_{q l}$ remains unchanged in the earlier category of 2 -adic $\psi^{2}$-modules, since it is still given by $T^{\infty} A$. To prove Lemma 7.10, we need:

Lemma 13.3. For a strictly nonlinear 2-adic Adams module A, each submodule is strictly nonlinear. Moreover, when $A$ is finitely generated over $\hat{\mathbb{Z}}_{2}$, each torsion-free quotient module is strictly nonlinear.

Proof. The first statement is clear, and we shall prove the second by working in the earlier category $\hat{\mathcal{N}}$ of 2 -adic $\psi^{2}$-modules that are $\psi^{2}$-pro-nilpotent. Let $0 \rightarrow \tilde{A} \rightarrow$ $A \rightarrow \bar{A} \rightarrow 0$ be a short exact sequence in $\hat{\mathcal{N}}$ with $A$ strictly nonlinear and finitely generated over $\hat{\mathbb{Z}}_{2}$ and with $\bar{A}$ torsion-free. To show that $\bar{A}$ is strictly nonlinear, it suffices to show that $\operatorname{Hom}_{\hat{\mathcal{N}}}(H, \bar{A})=0$ for each torsion-free quasilinear $H \in \hat{\mathcal{N}}$ that is finitely generated over $\hat{\mathbb{Z}}_{2}$. Since $\bar{A}$ is torsion-free, it now suffices to show that $\operatorname{Hom}_{\hat{\mathcal{N}}}(H, \bar{A})$ is finite for such $H$. Hence, since $\operatorname{Hom}_{\hat{\mathcal{N}}}(H, A)=0$ by strict nonlinearity, it suffices to show that $\operatorname{Ext}_{\hat{\mathcal{N}}}^{1}(H, \tilde{A})$ is finite for such $H$. This finiteness follows using the exact sequence

$$
0 \longrightarrow \operatorname{Hom}_{\hat{\mathcal{N}}}(H, \tilde{A}) \longrightarrow \operatorname{Hom}_{\hat{\mathcal{A}} b}(H, \tilde{A}) \longrightarrow \operatorname{Hom}_{\hat{\mathcal{A}} b}(H, \tilde{A}) \longrightarrow \operatorname{Ext}_{\hat{\mathcal{N}}}^{1}(H, \tilde{A}) \longrightarrow 0
$$

with $\operatorname{Hom}_{\hat{\mathcal{N}}}(H, \tilde{A})=0$ by strict nonlinearity, where $\hat{\mathcal{A}} b$ is the category of 2 -profinite abelian groups.

Proof of Lemma 7.10. This result follows easily from Definition 7.8 and Lemma 13.3. 
Proof of Lemma 7.9. By [8, Lemma 5.5], there is an exact sequence

$$
0 \longrightarrow \widetilde{K}^{1}\left(X / X^{3} ; \hat{\mathbb{Z}}_{2}\right) \longrightarrow \widetilde{K}^{1}\left(X ; \hat{\mathbb{Z}}_{2}\right) \longrightarrow H^{3}\left(X ; \hat{\mathbb{Z}}_{2}\right)
$$

of 2-adic Adams modules with $H^{3}\left(X ; \hat{\mathbb{Z}}_{2}\right)$ linear and $\widetilde{K}^{1}\left(X / X^{3} ; \hat{\mathbb{Z}}_{2}\right)$ torsion-free, where $X^{3}$ is the 3 -skeleton of $X$. Hence, it suffices to show that $\widetilde{K}^{1}\left(X / X^{3} ; \hat{\mathbb{Z}}_{2}\right)$ is strictly nonlinear with monic $\psi^{2}$. Since $H^{m}\left(X ; \hat{\mathbb{Z}}_{2}\right)=0$ for sufficiently large $m$, the map $\widetilde{K}^{1}\left(X / X^{3} ; \hat{\mathbb{Z}}_{2}\right) \rightarrow \widetilde{K}^{1}\left(X^{m} / X^{3} ; \hat{\mathbb{Z}}_{2}\right)$ is monic for such $m$. Thus by skeletal induction, the operator $\psi^{2}$ on $Q \otimes \widetilde{K}^{1}\left(X / X^{3} ; \hat{\mathbb{Z}}_{2}\right)$ is annihilated by the polynomial $f(x)=\left(x-2^{2}\right)\left(x-2^{3}\right) \ldots\left(x-2^{k}\right)$ for sufficiently large $k$. It follows that $Q \otimes \widetilde{K}^{1}\left(X / X^{3} ; \hat{\mathbb{Z}}_{2}\right)$ is the direct sum of the eigenspaces $E_{i}$ of $\psi^{2}$ with eigenvalues $2^{i}$ for $2 \leqslant i \leqslant k$, and hence $\psi^{2}$ is monic on $\widetilde{K}\left(X / X^{3} ; \hat{\mathbb{Z}}_{2}\right)$ as desired. Moreover, the projection to $E_{i}$ is given by the operator $f_{i}\left(\psi^{2}\right) / f_{i}\left(2^{i}\right)$ on $Q \otimes \widetilde{K}^{1}\left(X / X^{3} ; \hat{\mathbb{Z}}_{2}\right)$ where $f_{i}(x)=f(x) /\left(x-2^{i}\right)$. This implies that $2^{v} \widetilde{K}^{1}\left(X / X^{3} ; \hat{\mathbb{Z}}_{2}\right)$ is contained in $\bigoplus_{i=2}^{k} E_{i} \cap$ $\widetilde{K}^{1}\left(X / X^{3} ; \hat{\mathbb{Z}}_{2}\right)$ where $2^{v}$ is the highest power of 2 dividing an integer $f_{i}\left(2^{i}\right)$ for some $i$. Since the above direct sum is strictly nonlinear, so is $2^{v} \widetilde{K}^{1}\left(X / X^{3} ; \hat{\mathbb{Z}}_{2}\right)$ by Lemma 13.3 , and hence so is $\widetilde{K}^{1}\left(X / X^{3} ; \hat{\mathbb{Z}}_{2}\right)$.

\section{Proof of the realizability theorem for $\hat{L} M$}

We shall prove Theorem 8.5, giving a strict isomorphism $\hat{L} M \cong K_{C R}^{*}\left(\widetilde{\mathrm{Fib}} f ; \hat{\mathbb{Z}}_{2}\right)$ for a companion map $f: \Omega^{\infty} \tilde{\mathcal{E}} \bar{M} \rightarrow \Omega^{\infty} \tilde{\mathcal{E}} \bar{\rho} \bar{M}$ of a strong 2-adic Adams $\Delta$-module $M$. For this, it will suffice by Theorem 4.9 to obtain an isomorphism $\hat{\Lambda} M_{C} \cong$ $K^{*}\left(\widetilde{\mathrm{Fib}} f ; \hat{\mathbb{Z}}_{2}\right)$ of the complex components. We do this by adapting our proof of the corresponding odd primary result (Theorem 4.7) in [8]. First, to determine the 2 -adic $K$-cohomology of the loops on $\Omega^{\infty} \tilde{\mathcal{E}} \bar{M}$ or $\Omega^{\infty} \tilde{\mathcal{E}} \bar{\rho} \bar{M}$, we may replace Theorem 11.2 of $[8]$ by the following two theorems.

Theorem 14.1. If $X=\Omega^{\infty} E$ for a 1-connected spectrum $E$ with $H^{2}\left(E ; \hat{\mathbb{Z}}_{2}\right)=0$, with $K^{0}\left(E ; \hat{\mathbb{Z}}_{2}\right)=0$, and with $K^{1}\left(E ; \hat{\mathbb{Z}}_{2}\right)$ torsion-free, then $K^{1}\left(\Omega X ; \hat{\mathbb{Z}}_{2}\right)=0$ and $K^{0}\left(\Omega X ; \hat{\mathbb{Z}}_{2}\right)$ is torsion-free.

Proof. This follows from [6, Theorem 8.3].

Using notation and terminology of [7] for a 1-connected space $X$, we obtain an augmented 2 -adic $\psi^{2}$-module $\hat{Q} K^{1}\left(X ; \hat{\mathbb{Z}}_{2}\right) \downarrow H^{3}\left(X ; \hat{\mathbb{Z}}_{2}\right)$ representing the AtiyahHirzebruch map $K^{1}\left(X ; \hat{\mathbb{Z}}_{2}\right) \rightarrow H^{3}\left(X ; \hat{\mathbb{Z}}_{2}\right)$, and we have:

Theorem 14.2. If $X$ is a 1-connected $H$-space with $K^{1}\left(\Omega X ; \hat{\mathbb{Z}}_{2}\right)=0$ and $K^{0}(\Omega X$; $\left.\hat{\mathbb{Z}}_{2}\right)$ torsion-free, then $\sigma: U\left(\hat{Q} K^{1}\left(X ; \hat{\mathbb{Z}}_{2}\right) \downarrow H^{3}\left(X ; \hat{\mathbb{Z}}_{2}\right)\right) \cong K^{0}\left(\Omega X ; \hat{\mathbb{Z}}_{2}\right)$.

Proof. This follows from [7, Theorem 10.2].

When $X$ is $\Omega^{\infty} \tilde{\mathcal{E}} \bar{M}$ or $\Omega^{\infty} \tilde{\mathcal{E}} \bar{\rho} \bar{M}$, we shall determine $H^{3}\left(X ; \hat{\mathbb{Z}}_{2}\right)$ from the united 2adic $K$-cohomology of $X$. For any 1 -connected space $X$, we let $\alpha_{R}: \widetilde{K O}^{-1}\left(X ; \hat{\mathbb{Z}}_{2}\right) \rightarrow$ $H^{3}\left(X ; \hat{\mathbb{Z}}_{2}\right)$ be the homomorphism induced by the Postnikov section $K O \hat{\mathbb{Z}}_{2} \rightarrow$ 
$P^{4} K O \hat{\mathbb{Z}}_{2}$. Using the indecomposables $\hat{Q} K O^{*}\left(X ; \hat{\mathbb{Z}}_{2}\right)$ of Definition 2.7 and Remark 4.10, we have:

Lemma 14.3. If $X$ is a 1-connected space with $H^{2}\left(X ; \hat{\mathbb{Z}}_{2}\right)=0$, then $\alpha_{R}: \widetilde{K O}^{-1}(X$; $\left.\hat{\mathbb{Z}}_{2}\right) \rightarrow H^{3}\left(X ; \hat{\mathbb{Z}}_{2}\right)$ factors through $\hat{Q} K O^{-1}\left(X ; \hat{\mathbb{Z}}_{2}\right)$ and vanishes on the following subgroups: $\bar{\phi} \widetilde{K}^{-1}\left(X ; \hat{\mathbb{Z}}_{2}\right), \quad(\theta-2) \widetilde{K O}^{-1}\left(X ; \hat{\mathbb{Z}}_{2}\right), \quad\left(\theta-r B^{-2} c\right) \widetilde{K O}^{-5}\left(X ; \hat{\mathbb{Z}}_{2}\right)$, and $\left(\psi^{3}-9\right) \widetilde{K O}^{-1}\left(X ; \hat{\mathbb{Z}}_{2}\right)$.

Proof. The map $\alpha_{R}$ factors through $\hat{Q} K O^{-1}\left(X ; \hat{\mathbb{Z}}_{2}\right)$ by a suspension argument using the isomorphism $H^{3}\left(X ; \hat{\mathbb{Z}}_{2}\right) \cong H^{2}\left(\Omega X ; \hat{\mathbb{Z}}_{2}\right)$. Since $X$ is 1-connected with $H^{2}\left(X ; \hat{\mathbb{Z}}_{2}\right)$ $=0$, there is a natural isomorphism $H^{3}\left(X ; \hat{\mathbb{Z}}_{2}\right) \cong\left(\pi_{2}\left(\tau_{2} X\right)\right)^{\#}$ by [8, Lemma 11.4]. Thus, it suffices by naturality to prove the desired vanishing results when $X$ is $S^{2} \cup_{2^{k}} e^{3}$ for $k \geqslant 1$, and these results now follow from the elementary case $X=S^{3}$ since the collapsing map $S^{2} \cup_{2^{k}} e^{3} \rightarrow S^{3}$ induces epimorphisms of the cohomologies $\widetilde{K}^{-1}\left(-; \hat{\mathbb{Z}}_{2}\right), \widetilde{K O}^{-1}\left(-; \hat{\mathbb{Z}}_{2}\right)$, and $\widetilde{K O}^{-5}\left(-; \hat{\mathbb{Z}}_{2}\right)$.

For a 1-connected space $X$ with $H^{2}\left(X ; \hat{\mathbb{Z}}_{2}\right)=0$, the above $\alpha_{R}$ now induces a homomorphism $\bar{\alpha}_{R}: \operatorname{Lin}^{\Delta} \hat{Q} K_{\Delta}^{-1}\left(X ; \hat{\mathbb{Z}}_{2}\right) \rightarrow H^{3}\left(X ; \hat{\mathbb{Z}}_{2}\right)$ where $\hat{Q} K_{\Delta}^{-1}\left(X ; \hat{\mathbb{Z}}_{2}\right)$ is the 2-adic Adams $\Delta$-module of indecomposables given by Remark 4.10 and Definition 6.1, and where $\operatorname{Lin}^{\Delta}$ carries a 2 -adic Adams $\Delta$-module $M$ to the group

$$
\operatorname{Lin}^{\Delta} M=M_{R} /\left(\bar{\phi} M_{C}+(\theta-2) M_{R}+\left(\theta-r c^{\prime}\right) M_{H}+\left(\psi^{3}-9\right) M_{R}\right) .
$$

To determine $H^{3}\left(X ; \hat{\mathbb{Z}}_{2}\right)$ when $X$ is $\Omega^{\infty} \tilde{\mathcal{E}} \bar{M}$ or $\Omega^{\infty} \tilde{\mathcal{E}} \bar{\rho} \bar{M}$, we may replace Proposition 11.3 of $[\mathbf{8}]$ by:

Proposition 14.4. If $N$ is a torsion-free exact stable 2-adic Adams $\Delta$-module, then

$$
\bar{\alpha}_{R}: \operatorname{Lin}^{\Delta} \hat{Q} K_{\Delta}^{-1}\left(\Omega^{\infty} \tilde{\mathcal{E}} N ; \hat{\mathbb{Z}}_{2}\right) \cong H^{3}\left(\Omega^{\infty} \tilde{\mathcal{E}} N ; \hat{\mathbb{Z}}_{2}\right) .
$$

Proof. Since there is a stable isomorphism $\bar{\alpha}_{R}: K^{-1}\left(\tilde{\mathcal{E}} N ; \hat{\mathbb{Z}}_{2}\right) /\left(\psi^{3}-9\right) \cong H^{3}(\tilde{\mathcal{E}} N ;$ $\left.\hat{\mathbb{Z}}_{2}\right)$ by [10, Theorem 3.2] and [8, Lemma 11.4], the proposition follows using Theorem 6.7 and Lemma 4.11 .

For any $\theta$-pro-nilpotent 2-adic Adams $\Delta$-module $M$, we obtain a homomorphism $r: M^{C} \rightarrow \operatorname{Lin}^{\Delta} M$ of 2-adic Adams modules with $M^{C}$ as in Definition 7.6 and $\operatorname{Lin}^{\Delta} M$ linear. Such a homomorphism is called properly torsion-free $[\mathbf{7}, 4.5]$ when its source is torsion-free and its kernel is strictly nonlinear (see Definition 7.7). We shall need:

Lemma 14.5. If $M$ is a strong 2-adic Adams $\Delta$-module, then $r: M^{C} \rightarrow \operatorname{Lin}^{\Delta} M$ is properly torsion-free.

Proof. Since $M$ is strong, $M^{C}$ is torsion-free and $\operatorname{ker}\left(M^{C} \rightarrow \operatorname{Lin} M^{C}\right)$ is strictly nonlinear. Using the maps $r: \operatorname{Lin} M^{C} \rightarrow \operatorname{Lin}^{\Delta} M$ and $c: \operatorname{Lin}^{\Delta} M \rightarrow \operatorname{Lin} M^{C}$ with $c r=2$, we see that $2 \operatorname{ker}\left(M^{C} \rightarrow \operatorname{Lin}^{\Delta} M\right)$ is contained in $\operatorname{ker}\left(M^{C} \rightarrow \operatorname{Lin} M\right)$. Thus $\operatorname{ker}\left(M^{C} \rightarrow \operatorname{Lin}^{\Delta} M\right)$ is strictly nonlinear by Lemma 13.3 . 
As in [8, Section 11], for a strong 2 -adic Adams $\Delta$-module $M$ and a companion map $f$, we obtain a ladder of $p$-complete fiber sequences

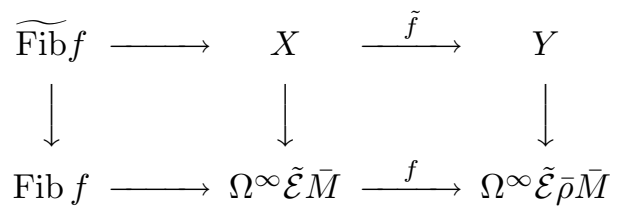

such that:

(i) $X$ and $Y$ satisfy the hypotheses of Theorems 14.1 and 14.2;

(ii) the vertical maps from $X$ and $Y$ are $K^{*}\left(-; \hat{\mathbb{Z}}_{2}\right)$-equivalences;

(iii) $H^{3}\left(Y ; \hat{\mathbb{Z}}_{2}\right)=0$ and the sequence $H^{3}\left(\Omega^{\infty} \tilde{\mathcal{E}} \bar{\rho} \bar{M} ; \hat{\mathbb{Z}}_{2}\right) \rightarrow H^{3}\left(\Omega^{\infty} \tilde{\mathcal{E}} \bar{M} ; \hat{\mathbb{Z}}_{2}\right) \rightarrow$ $H^{3}\left(X ; \hat{\mathbb{Z}}_{2}\right) \rightarrow 0$ is exact.

Lemma 14.6. There is a canonical isomorphism $H^{3}\left(X ; \hat{\mathbb{Z}}_{2}\right) \cong \operatorname{Lin}^{\Delta} M$.

Proof. Since $f^{*}: K_{C R}^{*}\left(\Omega^{\infty} \tilde{\mathcal{E}} \bar{\rho} \bar{M} ; \hat{\mathbb{Z}}_{2}\right) \rightarrow K_{C R}^{*}\left(\Omega^{\infty} \tilde{\mathcal{E}} \bar{M} ; \hat{\mathbb{Z}}_{2}\right)$ is equivalent to $\hat{L} \bar{d}$ : $\hat{L} \tilde{F} \bar{\rho} \bar{M} \rightarrow \hat{L} \tilde{F} \bar{M}$ for the $\theta$-resolution map $\bar{d}$, the homomorphism $f^{*}: H^{3}\left(\Omega^{\infty} \tilde{\mathcal{E}} \bar{\rho} \bar{M}\right.$; $\left.\hat{\mathbb{Z}}_{2}\right) \rightarrow H^{3}\left(\Omega^{\infty} \tilde{\mathcal{E}} \bar{M} ; \hat{\mathbb{Z}}_{2}\right)$ is equivalent to $\operatorname{Lin}^{\Delta} \bar{d}: \operatorname{Lin}^{\Delta} \tilde{F} \bar{\rho} \bar{M} \rightarrow \operatorname{Lin}^{\Delta} \tilde{F} \bar{M}$ by Proposition 14.4. Hence, there is an isomorphism of cokernels $H^{3}\left(X ; \hat{\mathbb{Z}}_{2}\right) \cong \operatorname{Lin}^{\Delta} M$.

Proof of Theorem 8.5. The proof of Theorem 4.7 in $[\mathbf{8}]$ is now easily adapted to give Theorem 8.5. In more detail, Propositions 11.5 and 11.6 of [8] remain valid in our setting using Lemmas 14.5 and 14.6 together with the short exact sequence

$$
0 \longrightarrow\left(\tilde{F} M^{C} \downarrow 0\right) \longrightarrow\left(\tilde{F} M^{C} \downarrow \operatorname{Lin}^{\Delta} M\right) \longrightarrow\left(M^{C} \downarrow \operatorname{Lin}^{\Delta} M\right) \longrightarrow 0
$$

induced by the $\theta$-resolution. Propositions 11.7 and 11.8 likewise remain valid, and thus $\hat{\Lambda} M^{C} \cong K^{*}\left(\widetilde{\mathrm{Fib}} f ; \hat{\mathbb{Z}}_{2}\right)$, so that Theorem 8.5 follows by Theorem 4.9.

\section{References}

[1] M. Bendersky, D.M. Davis and M. Mahowald, Stable geometric dimension of vector bundles over even-dimensional real projective spaces, Trans. Amer. Math. Soc. 358 (2006), 1585-1603.

[2] A.K. Bousfield, The localization of spaces with respect to homology, Topology 14 (1975), 133-150.

[3] A.K. Bousfield, The localization of spectra with respect to homology, Topology 18 (1979), 257-281.

[4] A.K. Bousfield, Uniqueness of infinite deloopings of $K$-theoretic spaces, Pacific J. Math. 129 (1987), 1-31.

[5] A.K. Bousfield, A classification of $K$-local spectra, J. Pure Appl. Algebra 66 (1990), 121-163.

[6] A.K. Bousfield, On $\lambda$-rings and the $K$-theory of infinite loop spaces, $K$-Theory 10 (1996), 1-30. 
[7] A.K. Bousfield, On $p$-adic $\lambda$-rings and the $K$-theory of $H$-spaces, Mathematisches Zeitschrift 223 (1996), 483-519.

[8] A.K. Bousfield, The $K$-theory localizations and $v_{1}$-periodic homotopy groups of $H$-spaces, Topology 38 (1999), 1239-1264.

[9] A.K. Bousfield, On the telescopic homotopy theory of spaces, Trans. Amer. Math. Soc. 353 (2000), 2391-2426.

[10] A.K. Bousfield, On the 2-primary $v_{1}$-periodic homotopy groups of spaces, Topology 44 (2005), 381-413.

[11] A.K. Bousfield, Kunneth theorems and unstable operations in 2-adic $K O$ cohomology, K-Theory, to appear.

[12] T. Bröcher and T. tom Dieck, Representations of Compact Lie Groups, Springer-Verlag, Berlin, 1985.

[13] D.M. Davis, Computing $v_{1}$-periodic homotopy groups of spheres and some compact Lie groups, Handbook of Algebraic Topology, I.M. James, ed., Elsevier, Amsterdam, 1995, pp. 93-1048.

[14] D.M. Davis, Representation types and 2-primary homotopy groups of certain compact Lie groups, Homology, Homotopy, and Applications 5 (2003), 297324.

[15] D.M. Davis and M. Mahowald, Some remarks on $v_{1}$-periodic homotopy groups, Adams Memorial Symposium on Algebraic Topology, London Mathematical Society Lecture Note Series, vol. 176, 1992, pp. 55-72.

[16] D.M. Davis and M. Mahowald, $v_{1}$-localizations of torsion spectra and spherically resolved spaces, Topology 32 (1993), 543-550.

[17] L. Hodgkin, On the $K$-theory of Lie groups, Topology 6 (1967), 1-36.

[18] N.J. Kuhn, Morava $K$-theories and infinite loop spaces, Lecture Notes in Mathematics, vol. 1370, Springer-Verlag, Berlin, 1989, pp. 243-257.

[19] S. Mac Lane, Categories for the Working Mathematician, Graduate Texts in Mathematics, vol. 5, Springer-Verlag, Berlin, 1971.

[20] M. Mahowald and R.D. Thompson, The $K$-theory localization of an unstable sphere, Topology 31 (1992), 133-141.

[21] H. Minami, On the $K$-theory of $S O(n)$, Osaka J. Math. 21 (1984), 789-808.

[22] L. Ribes and P. Zalesskii, Profinite Groups, Springer-Verlag, Berlin, 2000.

[23] R.M. Seymour, The real $K$-theory of Lie groups and homogeneous spaces, Quart. J. Math. Oxford 24 (1973), 7-30.

A.K. Bousfield bous@uic.edu

Department of Mathematics

University of Illinois at Chicago

Chicago, Illinois 60607

This article is available at http://intlpress.com/HHA/v9/n1/a14 